\title{
Structure of Essential Spectrum and Discrete Spectrum of the Energy Operator of Three-Magnon Systems in the Isotropic Ferromagnetic Non-Heisenberg Model with Spin One and Nearest-Neighbor Interactions
}

\author{
S. M. Tashpulatov \\ Institute of Nuclear Physics of Academy of Sciences of Republik of Uzbekistan, Tashkent, Uzbekistan \\ Email: sadullatashpulatov@yandex.ru, toshpul@mail.ru, toshpul@inp.uz
}

How to cite this paper: Tashpulatov, S.M. (2019) Structure of Essential Spectrum and Discrete Spectrum of the Energy Operator of Three-Magnon Systems in the Isotropic Ferromagnetic Non-Heisenberg Model with Spin One and Nearest-Neighbor Interactions. Journal of Applied Mathematics and Physics, 7, 874-899.

https://doi.org/10.4236/jamp.2019.74059

Received: October 18, 2018

Accepted: April 21, 2019

Published: April 24, 2019

Copyright (๑) 2019 by author(s) and Scientific Research Publishing Inc. This work is licensed under the Creative Commons Attribution-NonCommercial International License (CC BY-NC 4.0). http://creativecommons.org/licenses/by-nc/4.0/ (c) (7) (8) Open Access

\begin{abstract}
We consider a three-magnon system in the isotropic ferromagnetic NonHeisenberg model with spin one and with a coupling between nearest-neighbors. The structure of essential spectrum and discrete spectrum of the systems in a $v$-dimensional lattice are investigated. We obtain the lower and upper estimates for the number of three-magnon bound states of the system.
\end{abstract}

\section{Keywords}

Essential Spectrum, Discrete Spectrum, Three-Magnon Bound State, Two-Magnon Bound State, Non-Heisenberg Model

\section{Introduction}

Two-magnon systems have attracted the attention of many researchers. Probably, such systems were first discussed by Bethe [1] in the context of one-dimensional integer-valued lattices. Bethe proved that no more than one bound state (BS) of the system can exist in the case of one-dimensional isotropic ferromagnet. $\mathrm{N}$. Fukuda and M. Wortis [2] investigated the two-magnon systems in the one-dimensional Heisenberg ferromagnetic model and they have received the confirmation of Bethe results. Worts [3] examined the two-magnon system in a $d$-dimensional integer-valued lattice for an arbitrary $d$ and proved that in this case, the system has $0,1,2, \cdots, 2 d$ BSs. 
Majumdar [4] investigated the two-magnon system in a one-dimensional Heisenberg ferromagnet with a coupling between nearest and second nearest neighbors for the full quasi-momentum $\Lambda=\pi$. He found the spectrum and the BSs of the system numerically. In [5], such a system was examined for the case of a one-dimensional Heisenberg isotropic ferromagnet with a nearest- and second nearest-neighbor interactions for $\Lambda=\pi$ and $\Lambda=\frac{\pi}{2}$. The spectrum and the BSs of the system for these values of $\Lambda$ were studied with numerical methods. Gochev [6] considered the two-magnon system in a one-dimensional Heisenberg longitudinal ferromagnet with a coupling between nearest and second nearest neighbors for an arbitrary full quasi-momentum. He investigated the spectrum and the BSs of the system analytically.

The two-magnon systems in the anisotropic Heisenberg model with a nearest-neighbor interaction were addressed in [7]. The focus in [8] was on two-magnon systems in a one-dimensional anisotropic Heisenberg ferromagnet with a interaction between nearest and second nearest neighbors. The spectrum and the BSs of such systems were investigated for all values of the full quasi-momentum.

The usual starting point for theoretical studies of magnetically organized matter is the Heisenberg exchange Hamiltonian (with an arbitrary spin $s$ )

$$
H=J \sum_{m, \tau}\left(\boldsymbol{S}_{m} \boldsymbol{S}_{m+\tau}\right),
$$

where $J$ is the bilinear exchange interaction parameter for nearest-neighbor atoms; $S_{m}=\left(S_{m}^{x}, S_{m}^{y}, S_{m}^{z}\right)$ is the atomic spin operator of the $m$ th node of the $v$-dimensional integer-valued lattice $Z^{v}$, and $\tau$ denotes summation over the nearest neighbors. However, the actual isotropic spin exchange Hamiltonian with an arbitrary spin $s$ has the form [9]

$$
H=\sum_{m, \tau} \sum_{n=1}^{2 s} J_{n}\left(\boldsymbol{S}_{m} \boldsymbol{S}_{m+\tau}\right)^{n},
$$

where $J_{n}$ are the multipole exchange interaction parameters for nearest-neighbors atoms. Hamiltonian (2) coincides with Hamiltonian (1) only for $s=1 / 2$, while there are terms with higher powers of $\boldsymbol{S}_{m} \boldsymbol{S}_{m+\tau}$ up to $\left(\boldsymbol{S}_{m} \boldsymbol{S}_{m+\tau}\right)^{2 s}$ inclusive for $s>1 / 2$. These terms must be taken into account. Hamiltonian (2) is called the non-Heisenberg Hamiltonian.

Spectrum and BSs of two-magnon system in the non-Heisenberg ferromagnet with the bilinear and biquadratic exchange interactions were studied in works [10]-[17]. The spectrum and the BSs of two-magnon systems in a non-Heisenberg ferromagnet with coupling between nearest neighbors by bilinear and biquadratic interactions were investigated in [10] [11] [12] [13] [14]. Different methods, such as the Green's function method, the molecular field approximation method, the random phase approximation method, numerical methods, and the use of the creation and annihilation operators through the Holstein-Primakoff transformation, Dyson transformation, Dyson-Maleev transformation, Golghirch transformation, 
and others, were applied in these works. In [15] [16], the spectrum and the BSs of this system were investigated for the case of a one-dimensional non-Heisenberg ferromagnet with $s=1$ and with a coupling between second nearest and third nearest neighbors respectively. The values of the Hamiltonian parameters for which the BSs exist were found, and the energies of these BSs were calculated. In [17], the spectrum and the BSs of two-magnon system were investigated in a $v$-dimensional non-Heisenberg ferromagnet with $s=1$ and with a coupling between nearest neighbors.

In [18], the spectrum and the three-magnon BSs of three-magnon systems were investigated in a two-dimensional isotropic and anisotropic Heisenberg ferromagnet in bounded lattice with numerical methods.

In the work [19] [20] investigated the structure of essential spectrum and obtained the lower and upper estimates for the number of three-particle bound states (BS) of the energy operator of two-magnon system in a isotropic Heisenberg and Non-Heisenberg ferromagnet model with impurity in a $v$-dimensional lattice $Z^{v}$ with nearest-neighbor interactions.

The spectrum and BSs of two-magnon systems in a non-Heisenberg ferromagnet with coupling between nearest-neighbors by linear and biquadratic interactions were investigated in [17].

\section{Hamiltonian}

In this paper, we investigate the structure of essential spectrum and we obtain the lower and upper estimates for the number of three-magnon bound states of the energy operator of three-magnon system in a isotropic Non-Heisenberg ferromagnet model with spin one and nearest-neighbor interactions in a $v$-dimensional lattice $Z^{v}$.

In this case the component $S_{m}^{z}$ of spin operator $S_{m}$ take up the values $1,0,-1$, i.e. $S_{m}^{z} \varphi_{0}=\varphi_{0}$, or $S_{m}^{z} \varphi_{0}=0$, or $S_{m}^{z} \varphi_{0}=-\varphi_{0}$, where $\varphi_{0}$ be the vacuum vector. We consider these case the separately. From the beginning. We consider the case, when the spin component $S_{m}^{z}$ take up the value 1 .

The system Hamiltonian has the form

$$
H=-J \sum_{m, \tau}\left(\boldsymbol{S}_{m} \boldsymbol{S}_{m+\tau}\right)-J_{1} \sum_{m, \tau}\left(\boldsymbol{S}_{m} \boldsymbol{S}_{m+\tau}\right)^{2},
$$

acts in the symmetrical Fo'ck space $\mathcal{H}, S_{m}=\left(S_{m}^{x}, S_{m}^{y}, S_{m}^{z}\right)$ is the atomic spin $s=1$ operator in the node $m, J>0, J_{1}>0$ are the respective the bilinear and biquadratic exchange interaction parameters for nearest-neighbor atoms of the lattice, and $\tau$ denotes summation over the nearest neighbors. We set $S_{m}^{ \pm}=S_{m}^{x} \pm i S_{m}^{y}$, where $S_{m}^{-}$and $S_{m}^{+}$are the respective magnon creation and annihilation operators at the site $m$. Let $\varphi_{0}$ be the so-called vacuum vector, which is fully determined by the conditions $S_{m}^{+} \varphi_{0}=0$ and $S_{m}^{z} \varphi_{0}=\varphi_{0},\left\|\varphi_{0}\right\|=1$. The vectors $S_{m}^{-} S_{n}^{-} S_{l}^{-} \varphi_{0}$ describe the state of the system of three magnons located at the nodes $m, n$ and $l$. The vectors $S_{m}^{-} S_{n}^{-} S_{l}^{-} \varphi_{0}$ constitute an orthonormal system. We let $\mathcal{H}_{3}$ denote the Hilbert space spanned by these 
vectors. It is called the space of three-magnon states of the operator $H$. The space $\mathcal{H}_{3}$ is invariant under operator $H$. We let $H_{3}$ denote the restriction of the operator $H$ in the space $\mathcal{H}_{3}$.

Theorem 1. The space $\mathcal{H}_{3}$ is invariant with respect to the operator $H$. The operator $\mathrm{H}_{3}$ is a bounded self-adjoint operator. It generates the bounded self-adjoint operator $\bar{H}_{3}$, acting in the space $l_{2}\left(\left(Z^{v}\right)^{3}\right)$ according to the formula

$$
\begin{aligned}
& \left(\bar{H}_{3} f\right)(p, q, r) \\
& =-J \sum_{p, q, r, \tau}\left\{\left(\delta_{p, q+\tau}+\delta_{p, r+\tau}+\delta_{p+\tau, q}+\delta_{p+\tau, r}+\delta_{q, r+\tau}+\delta_{q+\tau, r}-6\right) f(p, q, r)\right. \\
& +\left(-\frac{1}{2} \delta_{p-\tau, q}-\frac{1}{2} \delta_{p-\tau, r}+1\right) f(p-\tau, q, r)+\left(-\frac{1}{2} \delta_{q-\tau, r}-\frac{1}{2} \delta_{p, q-\tau}+1\right) f(p, q-\tau, r) \\
& +\left(-\frac{1}{2} \delta_{p, r-\tau}-\frac{1}{2} \delta_{q, r-\tau}+1\right) f(p, q, r-\tau)+\left(-\frac{1}{2} \delta_{p+\tau, q}-\frac{1}{2} \delta_{p+\tau, r}+1\right) f(p+\tau, q, r) \\
& \left.+\left(-\frac{1}{2} \delta_{p, q+\tau}-\frac{1}{2} \delta_{q+\tau, r}+1\right) f(p, q+\tau, r)+\left(-\frac{1}{2} \delta_{p, r+\tau}-\frac{1}{2} \delta_{q, r+\tau}+1\right) f(p, q, r+\tau)\right\} \\
& -J_{1} \sum_{p, q, r, \tau}\left\{\left(-2 \delta_{p-\tau, q}-2 \delta_{p-\tau, r}-2 \delta_{p+\tau, q}-2 \delta_{p+\tau, r}-2 \delta_{q, r+\tau}-2 \delta_{q+\tau, r}-\frac{3}{2} \delta_{p, q+\tau} \delta_{q, r}\right.\right. \\
& -\frac{3}{2} \delta_{p, q+\tau} \delta_{p, r}-\frac{3}{2} \delta_{p, r+\tau} \delta_{p, q}-\frac{3}{2} \delta_{p+\tau, q} \delta_{q, r}-\frac{3}{2} \delta_{p+\tau, q} \delta_{p, r}-\frac{3}{2} \delta_{p, r+\tau} \delta_{p, q}+12 \\
& \left.+2 \delta_{p, q}+2 \delta_{q, r}+2 \delta_{p, r}\right) f(p, q, r)+\left(\frac{1}{2} \delta_{p, q+\tau}+\frac{1}{2} \delta_{p, r+\tau}+\frac{3}{2} \delta_{p, q+\tau} \delta_{p, r}+\delta_{p, q+\tau} \delta_{q, r}\right. \\
& \left.+\frac{3}{2} \delta_{p, r+\tau} \delta_{p, q}-\delta_{p, q}-\delta_{p, r}-2\right) f(p-\tau, q, r)+\left(\frac{1}{2} \delta_{q, r+\tau}+\frac{1}{2} \delta_{p+\tau, q}+\frac{3}{2} \delta_{p, r+\tau} \delta_{p, q}\right. \\
& \left.+\delta_{p+\tau, q} \delta_{p, r}+\frac{3}{2} \delta_{p+\tau, q} \delta_{q, r}-\delta_{p, q}-\delta_{p, r}-2\right) f(p, q-\tau, r)+\left(\frac{1}{2} \delta_{p+\tau, r}+\frac{1}{2} \delta_{q+\tau, r}\right. \\
& \left.+\frac{3}{2} \delta_{p+\tau, q} \delta_{q, r}+\delta_{p+\tau, r} \delta_{p, q}+\frac{3}{2} \delta_{p, q+\tau} \delta_{p, r}-\delta_{p, r}-\delta_{q, r}-2\right) f(p, q, r-\tau) \\
& +\left(\frac{1}{2} \delta_{p+\tau, q}+\frac{1}{2} \delta_{p+\tau, r}+\frac{3}{2} \delta_{p+\tau, q} \delta_{p, r}+\delta_{p+\tau, q} \delta_{q, r}+\frac{3}{2} \delta_{p+\tau, r} \delta_{p, q}-\delta_{p, q}\right. \\
& \left.-\delta_{p, r}-2\right) f(p+\tau, q, r)+\left(\frac{1}{2} \delta_{p, q+\tau}+\frac{1}{2} \delta_{q+\tau, r}+\frac{3}{2} \delta_{p, q+\tau} \delta_{q, r}+\delta_{p, q+\tau} \delta_{p, r}\right. \\
& \left.+\frac{3}{2} \delta_{p+\tau, r} \delta_{p, q}-\delta_{p, q}-\delta_{q, r}-2\right) f(p, q+\tau, r)+\left(\frac{1}{2} \delta_{p, r+\tau}+\frac{1}{2} \delta_{q, r+\tau}\right. \\
& \left.+\frac{3}{2} \delta_{p, q+\tau} \delta_{q, r}+\delta_{p, r+\tau} \delta_{p, q}+\frac{3}{2} \delta_{p+\tau, q} \delta_{p, r}-\delta_{p, r}-\delta_{q, r}-2\right) f(p, q, r+\tau) \\
& +\left(-\delta_{p, r+\tau} \delta_{p, q}+\delta_{p, q}\right) f(p-\tau, q-\tau, r)+\left(-\delta_{p, q+\tau} \delta_{p, r}+\delta_{p, r}\right) f(p-\tau, q, r-\tau) \\
& +\left(-\delta_{p+\tau, q} \delta_{q, r}+\delta_{q, r}\right) f(p, q-\tau, r-\tau) \\
& +\left(-\frac{3}{4} \delta_{p, r} \delta_{p, q+\tau}+\delta_{p, q+\tau}-\frac{3}{4} \delta_{q, r} \delta_{p, q+\tau}\right) f(p-\tau, q+\tau, r) \\
& +\left(-\frac{3}{4} \delta_{p, q} \delta_{p, r+\tau}+\delta_{p, r+\tau}-\frac{3}{4} \delta_{q, r} \delta_{p, q+\tau}\right) f(p-\tau, q, r+\tau)
\end{aligned}
$$




$$
\begin{aligned}
& +\left(-\frac{3}{4} \delta_{p, r} \delta_{p+\tau, q}+\delta_{p+\tau, q}-\frac{3}{4} \delta_{q, r} \delta_{p+\tau, q}\right) f(p+\tau, q-\tau, r) \\
& +\left(-\frac{3}{4} \delta_{p, q} \delta_{p, r+\tau}+\delta_{q, r+\tau}-\frac{3}{4} \delta_{p, r} \delta_{p+\tau, q}\right) f(p, q-\tau, r+\tau) \\
& +\left(-\frac{3}{4} \delta_{q, r} \delta_{p+\tau, q}+\delta_{p+\tau, r}-\frac{3}{4} \delta_{p, q} \delta_{p+\tau, r}\right) f(p+\tau, q, r-\tau) \\
& +\left(-\frac{3}{4} \delta_{p, q} \delta_{p+\tau, r}+\delta_{q+\tau, r}-\frac{3}{4} \delta_{p, r} \delta_{p, q+\tau}\right) f(p, q+\tau, r-\tau) \\
& +\left(-\delta_{p+\tau, r} \delta_{p, q}+\delta_{p, q}\right) f(p+\tau, q+\tau, r) \\
& +\left(-\delta_{p+\tau, q} \delta_{p, r}+\delta_{p, r}\right) f(p+\tau, q, r+\tau) \\
& \left.+\left(-\delta_{p, q+\tau} \delta_{q, r}+\delta_{q, r}\right) f(p, q+\tau, r+\tau)\right\},
\end{aligned}
$$

where $\delta_{k, j}$ is the Kronecker symbol. The operator $H_{3}$ acts on the vector $\psi \in \mathcal{H}_{3}$ according to the formula

$$
H_{3} \psi=\sum_{p, q, r}\left(\bar{H}_{3} f\right)(p, q, r) S_{p}^{-} S_{q}^{-} S_{r}^{-} \varphi_{0} .
$$

Proof. The proof is by direct calculation in which we use the well-known commutation relations between the operators $S_{m}^{+}, S_{p}^{-}$, and $S_{n}^{z}$ : $\left[S_{m}^{+}, S_{p}^{-}\right]=2 \delta_{m, p} S_{m}^{z}$, and $\left[S_{m}^{z}, S_{n}^{ \pm}\right]= \pm \delta_{m, n} S_{m}^{ \pm}$.

Lemma 1. The spectra of the operators $\mathrm{H}_{3}$ and $\bar{H}_{3}$ coincide.

Proof. Because $\mathrm{H}_{3}$ and $\bar{H}_{3}$ are bounded self-adjoint operators, it follows from the Weyl criterion that there exist a sequence of vectors $\psi_{n}$ such that $\psi_{n}=\sum_{p, q, r} f_{n}(p, q, r) S_{p}^{-} S_{q}^{-} S_{r}^{-} \varphi_{0},\left\|\psi_{n}\right\|=1$, and

$$
\lim _{n \rightarrow \infty}\left\|H_{3} \psi_{n}-\lambda \psi_{n}\right\|=0 \text {, }
$$

where $\lambda \in \sigma\left(H_{3}\right)$. On the other hand,

$$
\begin{aligned}
&\left\|H_{3} \psi_{n}-\lambda \psi_{n}\right\|^{2}=\left(H_{3} \psi_{n}-\lambda \psi_{n}, H_{3} \psi_{n}-\lambda \psi_{n}\right) \\
&= \sum_{p, q, r}\left\|\bar{H}_{3} f_{n}(p, q, r)-\lambda f_{n}(p, q, r)\right\|^{2} \\
& \times\left(\frac{1}{\sqrt{8-8 \delta_{p, q} \delta_{p, r}}} S_{p}^{-} S_{q}^{-} S_{r}^{-} \varphi_{0}, \frac{1}{\sqrt{8-8 \delta_{p, q} \delta_{p, r}}} S_{p}^{-} S_{q}^{-} S_{r}^{-} \varphi_{0}\right) \\
&=\left\|\bar{H}_{3} F_{n}-\lambda F_{n}\right\|^{2} \times\left(\frac{1}{8-8 \delta_{p, q} \delta_{p, r}} S_{p}^{+} S_{q}^{+} S_{r}^{+} S_{p}^{-} S_{q}^{-} S_{r}^{-} \varphi_{0}, \varphi_{0}\right) \\
&=\left\|\left(\bar{H}_{3}-\lambda\right) F_{n}\right\|^{2}\left(\varphi_{0}, \varphi_{0}\right)=\left\|\left(\bar{H}_{3}-\lambda\right) F_{n}\right\|^{2} \rightarrow 0, \\
& n \rightarrow \infty . \text { Here } F_{n}=\left(f_{n}(p, q, r)\right)_{p, q, r \in Z^{\prime}} \text { and } \\
&\left\|F_{n}\right\|^{2}=\sum_{p, q, r}\left|f_{n}(p, q, r)\right|^{2}=\left\|\psi_{n}\right\|^{2}=1 . \text { It follows that } \lambda \in \sigma\left(\bar{H}_{3}\right) . \text { Consequently, }
\end{aligned}
$$
$\sigma\left(H_{3}\right) \subset \sigma\left(\bar{H}_{3}\right)$. Conversely, let $\bar{\lambda} \in \sigma\left(\bar{H}_{3}\right)$. Again by the Weyl criterion, there then exist a sequence $F_{n}$ such that $\left\|F_{n}\right\|=\sqrt{\sum_{p, q, r}\left|f_{n}(p, q, r)\right|^{2}}=1$ and

$$
\left\|\bar{H}_{3} F_{n}-\bar{\lambda} F_{n}\right\| \rightarrow 0 \text {, }
$$


as $n \rightarrow \infty$.

Setting $\psi_{n}=\frac{1}{\sqrt{8-8 \delta_{p, q} \delta_{p, r}}} \sum_{p, q, r} f_{n}(p, q, r) S_{p}^{-} S_{q}^{-} S_{r}^{-} \varphi_{0}$, we have $\left\|\psi_{n}\right\|=\left\|F_{n}\right\|=1$ and $\left\|\bar{H}_{3} F_{n}-\bar{\lambda} F_{n}\right\|=\left\|H_{3} \psi_{n}-\bar{\lambda} \psi_{n}\right\|$. This, together with Formula (7) and the Weyl criterion, implies that $\bar{\lambda} \in \sigma\left(H_{3}\right)$, and hence $\sigma\left(\bar{H}_{3}\right) \subset \sigma\left(H_{3}\right)$. These two relations imply that $\sigma\left(H_{3}\right)=\sigma\left(\bar{H}_{3}\right)$.

We let $\mathcal{F}$ denote the Fourier transform:

$$
\mathcal{F}: l_{2}\left(\left(Z^{v}\right)^{3}\right) \rightarrow L_{2}\left(\left(T^{v}\right)^{3}\right) \equiv \tilde{\mathcal{H}}_{3},
$$

where $T^{v}$ is a $v$-dimensional torus with the normalized Lebesgue measure $d \lambda$ : $\lambda\left(T^{v}\right)=1$. We set $\tilde{H}_{3}=\mathcal{F} \bar{H}_{3} \mathcal{F}^{-1}$.

Theorem 2. The Fourier transformation transforms the operator $\bar{H}_{3}$ into the bounded self-adjoint operator $\tilde{H}_{3}$ acting in the space $\tilde{\mathcal{H}}_{3}$ according to the formula

$$
\begin{aligned}
& \left(\tilde{H}_{3} f\right)(\lambda, \mu, \gamma)=4\left(J-2 J_{1}\right) \sum_{i=1}^{v}\left[3-\cos \lambda_{i}-\cos \mu_{i}-\cos \gamma_{i}\right] f(\lambda, \mu, \gamma) \\
& -2\left(J-J_{1}\right) \int_{T^{v}} \sum_{i=1}^{v}\left[\cos \left(\lambda_{i}-s_{i}\right)+\cos \left(\mu_{i}-s_{i}\right)-\cos s_{i}-\cos \left(\lambda_{i}+\mu_{i}-s_{i}\right)\right] \\
& \times f(s, \lambda+\mu-s, \gamma) \mathrm{d} s-2\left(J-J_{1}\right) \int_{T^{v}} \sum_{i=1}^{v}\left[\cos \left(\lambda_{i}-s_{i}\right)+\cos \left(\gamma_{i}-s_{i}\right)-\cos s_{i}\right. \\
& \left.-\cos \left(\lambda_{i}+\gamma_{i}-s_{i}\right)\right] f(s, \mu, \lambda+\gamma-s) \mathrm{d} s-2\left(J-J_{1}\right) \int_{T^{v}} \sum_{i=1}^{v}\left[\cos \left(\mu_{i}-s_{i}\right)\right. \\
& \left.+\cos \left(\gamma_{i}-s_{i}\right)-\cos s_{i}-\cos \left(\mu_{i}+\gamma_{i}-s_{i}\right)\right] f(\lambda, s, \mu+\gamma-s) \mathrm{d} s \\
& -4 J_{1} \int_{T^{v}} \sum_{i=1}^{v}\left[1+\cos \left(\lambda_{i}+\mu_{i}\right)-\cos \lambda_{i}-\cos \mu_{i}\right] f(s, \lambda+\mu-s, \gamma) \mathrm{d} s \\
& -4 J_{1} \int_{T^{v}} \sum_{i=1}^{v}\left[1+\cos \left(\lambda_{i}+\gamma_{i}\right)-\cos \lambda_{i}-\cos \gamma_{i}\right] f(s, \mu, \lambda+\gamma-s) \mathrm{d} s \\
& -4 J_{1} \int_{T^{v}} \sum_{i=1}^{v}\left[1+\cos \left(\mu_{i}+\gamma_{i}\right)-\cos \mu_{i}-\cos \gamma_{i}\right] \times f(\lambda, s, \mu+\gamma-s) \mathrm{d} s \\
& +J_{1} \int_{T^{v}} \int_{T^{v}} \sum_{i=1}^{v}\left\{-6\left[\cos \left(\lambda_{i}-s_{i}\right)+\cos \left(\mu_{i}-t_{i}\right)+\cos \left(\lambda_{i}+\mu_{i}-s_{i}-t_{i}\right)\right]\right. \\
& +6\left[\cos \left(\lambda_{i}+\mu_{i}-t_{i}\right)+\cos \left(\mu_{i}-s_{i}-t_{i}\right)+\cos \left(\lambda_{i}+\mu_{i}-s_{i}\right)+\cos \left(\lambda_{i}-s_{i}-t_{i}\right)\right. \\
& \left.+\cos \left(\mu_{i}+\gamma_{i}-t_{i}\right)+\cos \left(\lambda_{i}+\gamma_{i}-s_{i}\right)\right]+2\left[3 \cos s_{i}+3 \cos t_{i}-2 \cos \left(s_{i}+t_{i}\right)\right. \\
& \left.-2 \cos \left(\lambda_{i}+\mu_{i}+\gamma_{i}-s_{i}\right)-2 \cos \left(\lambda_{i}+\mu_{i}+\gamma_{i}-t_{i}\right)\right]-3\left[\cos \left(\lambda_{i}-t_{i}\right)\right. \\
& +\cos \left(\mu_{i}-s_{i}\right)+\cos \left(\mu_{i}+\gamma_{i}-s_{i}-t_{i}\right)+\cos \left(\gamma_{i}-s_{i}\right)+\cos \left(\lambda_{i}+\gamma_{i}-s_{i}-t_{i}\right) \\
& \left.\left.+\cos \left(\gamma_{i}-t_{i}\right)\right]\right\} f(s, t, \lambda+\mu+\gamma-s-t) \mathrm{d} s \mathrm{~d} t . \\
& +8 \\
& \left.+\gamma_{i}\right)
\end{aligned}
$$

The following fact is important for further investigating the spectrum of the operator $\tilde{H}_{3}$. Let the full quasi-momentum of the three-magnon system, i.e. sum of quasi-momentum of each three magnons $x+y+z=\Lambda \in T^{v}$ be fixed. Let $L_{2}\left(\Gamma_{\Lambda}\right)$ be the space of functions that are quadratically integrable over the manifold $\Gamma_{\Lambda}=\{(x, y, z): x+y+z=\Lambda\}$. It is known [21] that the operator $\tilde{H}_{3}$ 
and space $\tilde{\mathcal{H}}_{3}$ can be expanded into the direct integrals $\tilde{H}_{3}=\oplus \int_{T^{\nu}} \tilde{H}_{3 \Lambda} \mathrm{d} \Lambda, \tilde{\mathcal{H}}_{3}=\oplus \int_{T^{\nu}} \tilde{\mathcal{H}}_{3 \Lambda} \mathrm{d} \Lambda$ of the operators $\tilde{H}_{3 \Lambda}$ and the spaces $\tilde{\mathcal{H}}_{3 \Lambda}$ such that the spaces $\tilde{\mathcal{H}}_{3 \Lambda}$ are invariant with respect to the operators $\tilde{H}_{3 \Lambda}$.

In the isotropic non-Heisenberg Ferromagnet model with spin $s=1$, the spectral properties of the considered operator of the energy of three-magnon systems are closely related to those of its two-magnon subsystems (see Formula (11)). We first study the spectrum and BSs of two-magnon systems.

\section{Two-Magnon Bound States}

The Hamiltoinian of a two-magnon subsystem also has form (3). We let $\mathcal{H}_{2}$ denote the space of two-magnon states of the operator $H$. We let $H_{2}$ denote the restriction of $H$ to the space $\mathcal{H}_{2}$.

Theorem 3. The space $\mathcal{H}_{2}$ is invariant with respect of the operator $H$. The operator $\mathrm{H}_{2}$ is a bounded self-adjoint operator. It generates the bounded self-adjoint operator $\bar{H}_{2}$, acting in the space $l_{2}\left(\left(Z^{v}\right)^{2}\right)$ according to the formula

$$
\begin{aligned}
& \left(\bar{H}_{2} f\right)(p, q)=-J \sum_{p, q, \tau}\left\{\left[\delta_{p, q+\tau}+\delta_{p+\tau, q}-4\right] f(p, q)-\frac{1}{2} \delta_{p-\tau, q} f(p-\tau, q)\right. \\
& -\frac{1}{2} \delta_{p, q-\tau} f(p, q-\tau)-\frac{1}{2} \delta_{p+\tau, q} f(p+\tau, q)-\frac{1}{2} \delta_{p, q+\tau} f(p, q+\tau) \\
& +f(p+\tau, q)+f(p-\tau, q)+f(p, q+\tau)+f(p, q-\tau)\} \\
& -J_{1} \sum_{p, q, \tau}\left\{\left[2 \delta_{p, q}-2 \delta_{p+\tau, q}-2 \delta_{p, q+\tau}+8\right] f(p, q)+\delta_{p, q+\tau} f(p-\tau, q+\tau)\right. \\
& +\delta_{p+\tau, q} f(p+\tau, q-\tau)-2 f(p-\tau, q)-2 f(p+\tau, q)+\delta_{p, q} f(p-\tau, q-\tau) \\
& +\delta_{p+\tau, q} f(p+\tau, q+\tau)+\frac{1}{2} \delta_{p, q+\tau} f(p-\tau, q)-\delta_{p, q} f(p-\tau, q)-2 f(p, q+\tau) \\
& -2 f(p, q-\tau)+\frac{1}{2} \delta_{p+\tau, q} f(p, q+\tau)-\delta_{p, q} f(p, q-\tau)+\frac{1}{2} \delta_{p, q+\tau} f(p, q+\tau) \\
& \left.+\frac{1}{2} \delta_{p+\tau, q} f(p+\tau, q)-\delta_{p, q} f(p, q+\tau)-\delta_{p, q} f(p+\tau, q)\right\} .
\end{aligned}
$$

The operator $H_{2}$ acts on the vector $\psi \in \mathcal{H}_{2}$ according to the formula

$$
H_{2} \psi=\sum_{p, q}\left(\bar{H}_{2} f\right)(p, q) S_{p}^{-} S_{q}^{-} \varphi_{0} .
$$

Lemma 2. The spectra of the operators $H_{2}$ and $\bar{H}_{2}$ coincide.

Theorem 4. The Fourier transformation transforms the operator $\bar{H}_{2}$ into the bounded self-adjoint operator $\tilde{H}_{2}$ acting in the space $\tilde{\mathcal{H}}_{2}$ according to the formula

$$
\left(\tilde{H}_{2} f\right)(x, y)=h(x, y) f(x, y)+\int_{T^{2}} h_{1}(x, y, t) f(t, x+y-t) \mathrm{d} t,
$$

where

$$
h(x, y)=8\left(J-2 J_{1}\right) \sum_{i=1}^{v}\left[1-\cos \frac{x_{i}+y_{i}}{2} \cos \frac{x_{i}-y_{i}}{2}\right],
$$




$$
\begin{aligned}
h_{1}(x, y, t)= & -4\left(J-J_{1}\right) \sum_{i=1}^{v}\left[\cos \frac{x_{i}-y_{i}}{2}-\cos \frac{x_{i}+y_{i}}{2}\right] \cos \left(\frac{x_{i}+y_{i}}{2}-t_{i}\right) \\
& -4 J_{1} \sum_{i=1}^{v}\left[1-2 \cos \frac{x_{i}+y_{i}}{2} \cos \frac{x_{i}-y_{i}}{2}+\cos \left(x_{i}+y_{i}\right)\right], x, y, t \in T^{v} .
\end{aligned}
$$

Let the full quasi-momentum of the two-magnon system, i.e. sum of quasi-momentum of each two magnons $x+y=\Lambda \in T^{v}$ be fixed. Let $L_{2}\left(\Gamma_{\Lambda}\right)$ be the space of functions that are quadratically integrable over the manifold $\Gamma_{\Lambda}=\{(x, y): x+y=\Lambda\}$. It is known [21] that the operator $\tilde{H}_{2}$ and space $\tilde{\mathcal{H}}_{2}$ can be expanded into the direct integrals $\tilde{H}_{2}=\oplus \int_{T^{\nu}} \tilde{H}_{2 \Lambda} \mathrm{d} \Lambda, \tilde{\mathcal{H}}_{2}=\oplus \int_{T^{\nu}} \tilde{\mathcal{H}}_{2 \Lambda} \mathrm{d} \Lambda$ of the operators $\tilde{H}_{2 \Lambda}$ and the spaces $\tilde{\mathcal{H}}_{2 \Lambda}$ such that the spaces $\tilde{\mathcal{H}}_{2 \Lambda}$ are invariant with respect to the operators $\tilde{H}_{2 \Lambda}$ and the operators $\tilde{H}_{2 \Lambda}$ act in the space $\tilde{\mathcal{H}}_{2 \Lambda}$ according to the formula

$$
\left(\tilde{H}_{2 \Lambda} f_{\Lambda}\right)(x)=h_{\Lambda}(x) f_{\Lambda}(x)+\int_{T^{v}} h_{1 \Lambda}(x, t) f_{\Lambda}(t) \mathrm{d} t,
$$

where $h_{\Lambda}(x)=h(x, \Lambda-x), h_{1 \Lambda}(x, t)=h_{1}(x, \Lambda-x, t)$ and $f_{\Lambda}(x)=f(x, \Lambda-x)$.

It is known that the continuous spectrum of the operator $\tilde{H}_{2 \Lambda}$ does not depend on the functions $h_{1 \Lambda}(x, t)$ and consists of the intervals

$$
G_{\Lambda}^{v}=\left[m_{\Lambda}^{v}, M_{\Lambda}^{v}\right] \text {, }
$$

where $m_{\Lambda}^{v}=\inf _{x \in T^{v}} h_{\Lambda}(x)$ and $M_{\Lambda}^{v}=\sup _{x \in T^{v}} h_{\Lambda}(x)$.

Definition 1. The eigenfunction $\varphi_{\Lambda} \in L_{2}\left(T^{v} \times T^{v}\right)$ of the operator $\tilde{H}_{2 \Lambda}$ corresponding to the eigenvalue $z_{\Lambda} \notin G_{\Lambda}^{v}$ is called the bound state (BS) of the operator $\tilde{H}_{2}$ with quasi-momentum $\Lambda$, and the quantity $z_{\Lambda}$ is called the energy of this BS.

We consider the operator $K_{\Lambda}(z)$, acting in the space $\tilde{\mathcal{H}}_{2 \Lambda}$ according to the formula

$$
K_{\Lambda}(z) f_{\Lambda}(x)=\int_{T^{v}} \frac{h_{1 \Lambda}(x, t)}{h_{\Lambda}(t)-z} f_{\Lambda}(t) \mathrm{d} t
$$

This operator is totally continuous in the space $\tilde{\mathcal{H}}_{2 \Lambda}$ for values of $z \notin G_{\Lambda}^{v}=\left[m_{\Lambda}^{v}, M_{\Lambda}^{v}\right]$.

Let $\Delta_{\Lambda}^{v}(z)=\operatorname{det} D_{\Lambda}^{v}(z)$, where

$$
D_{\Lambda}^{v}(z)=\left(\begin{array}{ccccc}
d_{1,1} & d_{1,2} & d_{1,3} & \cdots & d_{1, v+1} \\
d_{2,1} & d_{2,2} & d_{2,3} & \cdots & d_{2, v+1} \\
d_{3,1} & d_{3,2} & d_{3,3} & \cdots & d_{3, v+1} \\
\vdots & \vdots & \vdots & \ddots & \vdots \\
d_{v, 1} & d_{v, 2} & d_{v, 3} & \cdots & d_{v, v+1} \\
d_{v+1,1} & d_{v+1,2} & d_{v+1,3} & \cdots & d_{v+1, v+1}
\end{array}\right),
$$

and

$$
\begin{gathered}
d_{1,1}=1-4 J_{1} \int_{T^{v}} \frac{g_{\Lambda}(s) \mathrm{d} s}{h_{\Lambda}(s)-z}, \\
d_{1, k+1}=-4\left(J-J_{1}\right) \int_{T^{v}} \frac{\xi_{\Lambda_{k}}\left(s_{k}\right) \mathrm{d} s}{h_{\Lambda}(s)-z}, k=\overline{1, v},
\end{gathered}
$$




$$
\begin{gathered}
d_{k+1,1}=-4 J_{1} \int_{T V} \frac{\eta_{\Lambda_{k}}\left(s_{k}\right) g_{\Lambda}(s) \mathrm{d} s}{h_{\Lambda}(s)-z}, k=\overline{1, v}, \\
d_{k+1, k+1}=1-4\left(J-J_{1}\right) \int_{T^{v}} \frac{\eta_{\Lambda_{k}}\left(s_{k}\right) \xi_{\Lambda_{k}}\left(s_{k}\right) \mathrm{d} s}{h_{\Lambda}(s)-z}, k=\overline{1, v}, \\
d_{k+1, i+1}=-4\left(J-J_{1}\right) \int_{T^{v}} \frac{\eta_{\Lambda_{k}}\left(s_{k}\right) \xi_{\Lambda_{i}}\left(s_{i}\right) \mathrm{d} s}{h_{\Lambda}(s)-z}, k=\overline{1, v}, i=\overline{1, v}, k \neq i .
\end{gathered}
$$

In these formulas,

$$
\begin{gathered}
g_{\Lambda}(s)=\sum_{k=1}^{v}\left[1+\cos \Lambda_{k}-2 \cos \frac{\Lambda_{k}}{2} \cos \left(\frac{\Lambda_{k}}{2}-s_{k}\right)\right], \\
\xi_{\Lambda_{k}}\left(s_{k}\right)=\cos \left(\frac{\Lambda_{k}}{2}-s_{k}\right)-\cos \frac{\Lambda_{k}}{2}, \quad \eta_{\Lambda_{k}}\left(s_{k}\right)=\cos \left(\frac{\Lambda_{k}}{2}-s_{k}\right), k=\overline{1, v} .
\end{gathered}
$$

Lemma 3. A number $z_{0} \notin G_{\Lambda}^{v}$ be an eigenvalue of the operator $\tilde{H}_{2 \Lambda}$ if and only if it is a zero of the function $D_{\Lambda}^{v}(z)$, i.e. $D_{\Lambda}^{v}\left(z_{0}\right)=0$.

Proof. In the case under consideration, the equation for the eigenvalues is an integral equation with a degenerate kernel. It is therefore equivalent to a system of linear homogeneous algebraic equations. It is known that such a system has a nontrivial solution if and only if its determinant is equal to zero. In this case, the determinant of this linear homogeneous algebraic system is equal to function $\Delta_{\Lambda}^{v}(z)$.

Theorem 5. Let $J=2 J_{1}$ and $v$ be arbitrary. Then the operator $\tilde{H}_{2}$ has two BSs $\varphi_{1}$ and $\varphi_{2}$ (not taking the order of the energy degeneration into account) with the energy values $z_{1}=-2 J_{1}, z_{2}=-(4 v+2) J_{1}-4 J_{1} \sum_{i=1}^{v} \cos \Lambda_{i}$, and $z_{1}$ is degenerate $v$ times, while $z_{2}$ is not degenerate, $z_{i}<m_{\Lambda}^{v}, i=1,2$, for all $\Lambda \in T^{v}$, i.e. the energy values of these BSs lie below the continuous spectrum domain of the operator $\tilde{H}_{2}$.

Proof. If $J=2 J_{1}$, then $h_{\Lambda}(s) \equiv 0$, and

$$
\Delta_{\Lambda}^{v}(z)=\left(1+\frac{2 J_{1}}{z}\right)^{v}\left\{\left(1+\frac{2 J_{1}}{z}\right)\left[1+\frac{4 J_{1}}{z} \sum_{i=1}^{v}\left(1+\cos \Lambda_{i}\right)\right]-\frac{16 J_{1}^{2}}{z^{2}} \sum_{i=1}^{v} \cos ^{2} \frac{\Lambda_{i}}{2}\right\} .
$$

Solving the equation $\Delta_{\Lambda}^{v}(z)=0$, we prove the theorem.

Let $\tilde{\pi}=(\pi, \pi, \cdots, \pi) \in T^{v}$.

Theorem 6. Let $\Lambda=\tilde{\pi}$ and $J \neq J_{1}$. Then the operator $\tilde{H}_{2}$ has only one BS $\varphi$ with the energy value $z=8 v\left(J-2 J_{1}\right)-2\left(J-J_{1}\right)$, and this energy level is degenerate $v$ times. In addition, if $J>J_{1}$, then $z<m_{\Lambda}^{v}$, and if $J<J_{1}$, then $z>M_{\Lambda}^{v}$. When $J=J_{1}$, this BS vanishes because it is incorporated into the continuous spectrum.

Proof. The proof of this theorem is based on the equality $h_{\Lambda}(x)=8 v\left(J-2 J_{1}\right)$ with $\Lambda=\tilde{\pi}$ and also on the corresponding form of the determinant $\Delta_{\Lambda}^{v}(z)=\left(1-\frac{2\left(J-J_{1}\right)}{8 v\left(J-2 J_{1}\right)-z}\right)^{v}$.

From Theorem 5 and 6 and later is obviously, what the spectrum of the 
Hamiltonian $\mathcal{H}$ by different value of $v$ differ from one another.

In the case where $v=1$, the change of the energy spectrum of the operator $\tilde{H}_{2}$ is described by the following theorems.

\section{Theorem 7.}

1) Let $J<J_{1}$ and $\left.\Lambda \in\right] 0, \pi[$ or $\Lambda \in] \pi, 2 \pi[$.

a) If respectively $\cos \frac{\Lambda}{2}>-\frac{J-J_{1}}{2 J_{1}}$ or $\cos \frac{\Lambda}{2}<\frac{J-J_{1}}{2 J_{1}}$, then the operator $\tilde{H}_{2}$ has two BSs $\varphi_{1}$ and $\varphi_{2}$ with the corresponding energy values $z_{i}<m_{\Lambda}^{1}, i=1,2$.

b) If respectively $\cos \frac{\Lambda}{2} \leq-\frac{J-J_{1}}{2 J_{1}}$ or $\cos \frac{\Lambda}{2} \geq \frac{J-J_{1}}{2 J_{1}}$, then the operator $\tilde{H}_{2}$ has only one BS $\varphi_{1}$ with the energy value $z_{1}$, and $z_{1}<m_{\Lambda}^{1}$.

2) Let $J=J_{1}$ and $\left.\Lambda \in\right] 0, \pi[$ or $\Lambda \in] \pi, 2 \pi[$.

a) If respectively $0<\Lambda<\alpha_{1}$ or $\alpha_{2}<\Lambda<2 \pi$, then the operator $\tilde{H}_{2}$ has only one BS $\varphi$ with the energy value $z<m_{\Lambda}^{1}$.

b) If $\Lambda \in\left[\alpha_{1}, \pi[U] \pi, \alpha_{2}\left[\right.\right.$, then the operator $\tilde{H}_{2}$ has no BS. Above, $\alpha_{1} \approx 100^{\circ}, \alpha_{2} \approx 260^{\circ}$, and $z=-8 J_{1}-\frac{8 J_{1} \cos ^{2} \frac{\Lambda}{2}\left(1+2 \sqrt{3+\cos ^{2} \frac{\Lambda}{2}}\right)}{3}$.

3) Let $J_{1}<J<2 J_{1}$ and $\left.\Lambda \in\right] 0, \pi[$ or $\Lambda \in] \pi, 2 \pi[$.

a) If respectively $\cos \frac{\Lambda}{2} \leq \frac{J-J_{1}}{2 J_{1}}$ or $\cos \frac{\Lambda}{2} \geq-\frac{J-J_{1}}{2 J_{1}}$, then the operator $\tilde{H}_{2}$ has two BSs $\varphi_{1}$ and $\varphi_{2}$ with the corresponding energy values $z_{1}<m_{\Lambda}^{1}$, and $z_{2}>M_{\Lambda}^{1}$.

b) If respectively $\cos \frac{\Lambda}{2}>\frac{J-J_{1}}{2 J_{1}}$ or $\cos \frac{\Lambda}{2}<-\frac{J-J_{1}}{2 J_{1}}$, then the operator $\tilde{H}_{2}$ has three BSs $\varphi_{1}, \varphi_{2}$, and $\varphi_{3}$ with the corresponding energy values $z_{1}<m_{\Lambda}^{1}$, and $z_{i}>M_{\Lambda}^{1}, i=2,3$.

4) Let $2 J_{1}<J<3 J_{1}$ and $\left.\Lambda \in\right] 0, \pi[$ or $\Lambda \in] \pi, 2 \pi[$.

a) If respectively $\cos \frac{\Lambda}{2}>\frac{J-J_{1}}{2 J_{1}}$ or $\cos \frac{\Lambda}{2}<-\frac{J-J_{1}}{2 J_{1}}$, then the operator $\tilde{H}_{2}$ has two BSs $\varphi_{1}$ and $\varphi_{2}$ with the corresponding energy values $z_{i}<m_{\Lambda}^{1}, i=1,2$.

b) If respectively $\cos \frac{\Lambda}{2} \leq \frac{J-J_{1}}{2 J_{1}}$ or $\cos \frac{\Lambda}{2} \geq-\frac{J-J_{1}}{2 J_{1}}$, then the operator $\tilde{H}_{2}$ has only BS $\varphi_{1}$ with the energy value $z_{1}<m_{\Lambda}^{1}$. In this case, the second BS vanishes because it is incorporated into the continuous spectrum.

5) Let $J=3 J_{1}$ and $\Lambda \neq 0$. Then the operator $\tilde{H}_{2}$ has only one BS $\varphi$ with the energy value $z=4 J_{1}\left(1-\cos ^{2} \frac{\Lambda}{2}\right)<m_{\Lambda}^{1}$.

6) Let $J>3 J_{1}$ and $\Lambda \neq 0$. Then the operator $\tilde{H}_{2}$ has two BSs $\varphi_{1}$ and $\varphi_{2}$ with the corresponding energy values $z_{1}<m_{\Lambda}^{1}$, and $z_{2}>M_{\Lambda}^{1}$.

In the case where $v=1$ and $\Lambda=0$, the change of the energy spectrum is described by the following theorem. 


\section{Theorem 8.}

1) If $J<J_{1}$ and $\Lambda=0$, then the operator $\tilde{H}_{2}$ has two BSs $\varphi_{1}$ and $\varphi_{2}$ with the corresponding energy values $z_{i}<m_{\Lambda}^{1}, i=1,2$.

2) If $J=J_{1}$ and $\Lambda=0$, then the operator $\tilde{H}_{2}$ has only one BS $\varphi$ with the energy value $z=-\frac{64}{3} J_{1}<m_{\Lambda}^{1}$.

3) If $J_{1}<J<2 J_{1}$ and $\Lambda=0$, then the operator $\tilde{H}_{2}$ has two BSs $\varphi_{1}$ and $\varphi_{2}$ with the corresponding energy values $z_{1}<m_{\Lambda}^{1}$ and $z_{2}>M_{\Lambda}^{1}$.

4) If $2 J_{1}<J<3 J_{1}$ and $\Lambda=0$, then the operator $\tilde{H}_{2}$ has two BSs $\varphi_{1}$ and $\varphi_{2}$ with the corresponding energy values $z_{i}<m_{\Lambda}^{1}, i=1,2$.

5) If $J=3 J_{1}$ and $\Lambda=0$, then the operator $\tilde{H}_{2}$ has no BS.

6) If $J>3 J_{1}$ and $\Lambda=0$, then the operator $\tilde{H}_{2}$ has only one BS $\varphi$ with the energy value $z>M_{\Lambda}^{1}$.

A sketch proof of Theorems 7-8 is given below. In the case under consideration, the equation for the eigenvalues is an integral equation with a degenerate kernel. It is therefore equivalent to a system of linear homogeneous algebraic equations. It is known that such a system has a nontrivial solution if and only if its determinant is equal to zero. In this case, the equation $\Delta_{\Lambda}^{1}(z)=0$ it therefore equivalent to the equation stating that the determinant of the system is zero. Expressing all integrals in the equation $\Delta_{\Lambda}^{1}(z)=0$, through the integral $J^{\star}(z)=\int_{T} \frac{\mathrm{d} t}{h_{\Lambda}(t)-z}$, we find that the equation $\Delta_{\Lambda}^{1}(z)=0$ is equivalent to the equation

$$
\begin{aligned}
J^{\star}(z)= & \left\{8\left(J-2 J_{1}\right)\left(J-5 J_{1}\right) \cos ^{2} \frac{\Lambda}{2}-\left(J-J_{1}\right)\left[z-8\left(J-2 J_{1}\right)\right]\right\} \\
& \times\left\{128 J_{1}\left(J-2 J_{1}\right)^{2} \cos ^{4} \frac{\Lambda}{2}+8\left(J-2 J_{1}\right)\left(J+J_{1}\right)\right. \\
& \left.\times \cos ^{2} \frac{\Lambda}{2}\left[z-8\left(J-2 J_{1}\right)\right]+\left(J-J_{1}\right)\left[z-8\left(J-2 J_{1}\right)\right]^{2}\right\}^{-1} .
\end{aligned}
$$

Because $\frac{1}{h_{\Lambda}(t)-z}$ is a continuous function for $z \notin\left[m_{\Lambda}^{1} ; M_{\Lambda}^{1}\right]$ and $\left[J^{\star}(z)\right]^{\prime}=\int_{T} \frac{\mathrm{d} t}{\left[h_{\Lambda}(t)-z\right]^{2}}>0$, the function $J^{\star}(z)$ is an increasing function of $z$ for $z \notin\left[m_{\Lambda}^{1} ; M_{\Lambda}^{1}\right]$. Moreover, $J^{\star}(z) \rightarrow 0$ as $z \rightarrow-\infty, J^{\star}(z) \rightarrow+\infty$ as $z \rightarrow m_{\Lambda}^{1}-0, \quad J^{\star}(z) \rightarrow-\infty \quad$ as $\quad z \rightarrow M_{\Lambda}^{1}+0 \quad$ and $\quad J^{\star}(z) \rightarrow 0$ as $z \rightarrow+\infty$. Analysis of Equation (13) outside the set $G_{\Lambda}=\left[m_{\Lambda}^{1} ; M_{\Lambda}^{1}\right]$, leads to the proof of Theorems 7-8.

The energy spectrum in the case where $v=2$ for the full quasi momenta of the form $\Lambda=\left(\Lambda_{1}, \Lambda_{2}\right)=\left(\Lambda_{0}, \Lambda_{0}\right)$ is described below. It is easy to see that if the parameters $J, J_{1}$, and $\Lambda_{0}$ satisfy the conditions of Theorems 7-8, the statements of the theorems are true. Only one additional BS $\tilde{\varphi}$ appears, whose energy value is $\tilde{z}$, moreover $\tilde{z}<m_{\Lambda}^{2}\left(\tilde{z}>M_{\Lambda}^{2}\right)$, if $J>J_{1}\left(J<J_{1}\right)$. If $J=J_{1}$, 
the operator $\tilde{H}_{2}$ has no additional BS.

The proof of this statements is based on the fact that if $v=2$ and $\Lambda=\left(\Lambda_{0}, \Lambda_{0}\right)$, then the function $\Delta_{\Lambda}^{2}(z)$ has the form

$$
\Delta_{\Lambda}^{2}(z)=\left[1-2\left(J-J_{1}\right) \int_{T^{2}} \frac{\left[\cos \left(\frac{\Lambda_{0}}{2}-t_{1}\right)-\cos \left(\frac{\Lambda_{0}}{2}-t_{2}\right)\right]^{2} \mathrm{~d} t_{1} \mathrm{~d} t_{2}}{h_{\Lambda}\left(t_{1}, t_{2}\right)-z}\right] \Psi_{\Lambda}(z),
$$

where

$$
\begin{aligned}
& \Psi_{\Lambda}(z)=\left\{1-8 J_{1} \int_{T^{2}} \frac{1+\cos \Lambda_{0}-\cos \frac{\Lambda_{0}}{2}\left[\cos \left(\frac{\Lambda_{0}}{2}-t_{1}\right)+\cos \left(\frac{\Lambda_{0}}{2}-t_{2}\right)\right]}{h_{\Lambda}\left(t_{1}, t_{2}\right)-z} \mathrm{~d} t\right\} \\
& \times\left[1-4\left(J-J_{1}\right) \int_{T^{2}} \frac{\cos \left(\frac{\Lambda_{0}}{2}-t_{1}\right)\left[\cos \left(\frac{\Lambda_{0}}{2}-t_{1}\right)+\cos \left(\frac{\Lambda_{0}}{2}-t_{2}\right)-2 \cos \frac{\Lambda_{0}}{2}\right]}{h_{\Lambda}\left(t_{1}, t_{2}\right)-z} \mathrm{~d} t\right] \\
& -64\left(J-J_{1}\right) J_{1} \int_{T^{2}} \frac{\left\{1+\cos \Lambda_{0}-\cos \frac{\Lambda_{0}}{2}\left[\cos \left(\frac{\Lambda_{0}}{2}-t_{1}\right)+\cos \left(\frac{\Lambda_{0}}{2}-t_{2}\right)\right]\right\} \cos \left(\frac{\Lambda_{0}}{2}-t_{1}\right)}{h_{\Lambda}\left(t_{1}, t_{2}\right)-z} \mathrm{~d} t_{1} \mathrm{~d} t_{2} \\
& \times \int_{T^{2}} \frac{\cos \left(\frac{\Lambda_{0}}{2}-t_{1}\right)-\cos \frac{\Lambda_{0}}{2}}{h_{\Lambda}\left(t_{1}, t_{2}\right)-z} \mathrm{~d} t_{1} \mathrm{~d} t_{2} .
\end{aligned}
$$

The equation $\Delta_{\Lambda}^{2}(z)=0$ is therefore equivalent to the equation

$$
1-2\left(J-J_{1}\right) \int_{T^{2}} \frac{\left[\cos \left(\frac{\Lambda_{0}}{2}-t_{1}\right)-\cos \left(\frac{\Lambda_{0}}{2}-t_{2}\right)\right]^{2} \mathrm{~d} t_{1} \mathrm{~d} t_{2}}{h_{\Lambda}\left(t_{1}, t_{2}\right)-z}=0
$$

and

$$
\Psi_{\Lambda}(z)=0 .
$$

It is easy to see that Equation (15) has a unique solution $\tilde{z}<m_{\Lambda}^{2}$ if $J>J_{1}$; if $J<J_{1}$, this solution satisfies the condition $\tilde{z}>M_{\Lambda}^{2}$. If $J=J_{1}$, Equation (15) has no solution. Expressing the integrals in Equation (16) through the integral $J^{\star}(z)=\int_{T^{2}} \frac{\mathrm{d} t_{1} \mathrm{~d} t_{2}}{h_{\Lambda}\left(t_{1}, t_{2}\right)-z}$, we obtain an equation of the form

$$
\eta_{\Lambda}(z) J^{\star}(z)=\xi_{\Lambda}(z)
$$

where

$$
\begin{aligned}
& \qquad \eta_{\Lambda}(z)=\left(J-J_{1}\right) \tilde{z}^{2}+16\left(J-2 J_{1}\right)\left(J+J_{1}\right) \cos ^{2} \frac{\Lambda_{0}}{2} \tilde{z}+512 J_{1}\left(J-2 J_{1}\right) \cos ^{4} \frac{\Lambda_{0}}{2}, \\
& \text { and } \xi_{\Lambda}(z)=16\left(J-2 J_{1}\right)\left(J-5 J_{1}\right) \cos ^{2} \frac{\Lambda_{0}}{2}-\left(J-J_{1}\right) \tilde{z} . \text { In this case, }
\end{aligned}
$$


$\tilde{z}=z-16\left(J-2 J_{1}\right)$. In turn, for $\eta_{\Lambda}(z) \neq 0$, the latter equation is equivalent to the equation of the form

$$
J^{\star}(z)=\frac{\xi_{\Lambda}(z)}{\eta_{\Lambda}(z)} .
$$

Analyzing Equation (17) outside the set $G_{\Lambda}^{2}$ and taking into account that the function $J^{\star}(z)$ is monotonic for $z \notin\left[m_{\Lambda}^{2} ; M_{\Lambda}^{2}\right]$, we obtain statements similar to the statements in Theorems 7-8.

For all other quasi momenta $\Lambda=\left(\Lambda_{1}, \Lambda_{2}\right), \Lambda_{1} \neq \Lambda_{2}$, there exist sets $R_{j}, j=\overline{0,5}$, of the parameters $J, J_{1}$, and $\Lambda$ such that in every set $R_{j}$ the operator $\tilde{H}_{2}$ has exactly $j$ BSs (taking the energy degeneration order into account) with the corresponding energy values $z_{k} \notin G_{\Lambda}^{2}, k=\overline{1,5}$.

Indeed, in this case and for $v=2$, the function $\Delta_{\Lambda}^{2}(z)$ has the form

$$
\Delta_{\Lambda}^{2}(z)=\operatorname{det} D_{\Lambda}^{2}(z)
$$

where $D_{\Lambda}^{2}(z)=\left(\begin{array}{ccc}a_{1} & a_{2} & a_{3} \\ b_{1} & b_{2} & b_{3} \\ c_{1} & c_{2} & c_{3}\end{array}\right)$, and $a_{1}=1-4 J_{1} \int_{T^{2}} \frac{g_{\Lambda}(s) \mathrm{d} s}{h_{\Lambda}(s)-z}$,

$a_{k+1}=-4\left(J-J_{1}\right) \int_{T^{2}} \frac{f_{\Lambda_{k}}\left(s_{k}\right) \mathrm{d} s}{h_{\Lambda}(s)-z}, k=1,2 ; \quad b_{1}=-4 J_{1} \int_{T^{2}} \frac{\eta_{\Lambda_{1}}\left(s_{1}\right) g_{\Lambda}(s) \mathrm{d} s}{h_{\Lambda}(s)-z}$,

$b_{2}=1-4\left(J-J_{1}\right) \int_{T^{2}} \frac{\eta_{\Lambda_{1}}\left(s_{1}\right) f_{\Lambda_{1}}\left(s_{1}\right) \mathrm{d} s}{h_{\Lambda}(s)-Z}, \quad b_{3}=-4\left(J-J_{1}\right) \int_{T^{2}} \frac{\eta_{\Lambda_{1}}\left(s_{1}\right) f_{\Lambda_{2}}\left(s_{2}\right) \mathrm{d} s}{h_{\Lambda}(s)-Z}$,

$c_{1}=-4 J_{1} \int_{T^{2}} \frac{\eta_{\Lambda_{2}}\left(s_{2}\right) g_{\Lambda}(s) \mathrm{d} s}{h_{\Lambda}(s)-z}, \quad c_{2}=-4\left(J-J_{1}\right) \int_{T^{2}} \frac{\eta_{\Lambda_{2}}\left(s_{2}\right) f_{\Lambda_{1}}\left(s_{1}\right) \mathrm{d} s}{h_{\Lambda}(s)-z}$,

$c_{3}=1-4\left(J-J_{1}\right) \int_{T^{2}} \frac{\eta_{\Lambda_{2}}\left(s_{2}\right) f_{\Lambda_{2}}\left(s_{2}\right) \mathrm{d} s}{h_{\Lambda}(s)-z}$.

In these formulas, $g_{\Lambda}(s)=\sum_{k=1}^{2}\left[1+\cos \Lambda_{k}-2 \cos \frac{\Lambda_{k}}{2} \cos \left(\frac{\Lambda_{k}}{2}-s_{k}\right)\right]$,

$f_{\Lambda_{k}}\left(s_{k}\right)=\cos \left(\frac{\Lambda_{k}}{2}-s_{k}\right)-\cos \frac{\Lambda_{k}}{2}, k=1,2, \eta_{\Lambda_{k}}\left(s_{k}\right)=\cos \left(\frac{\Lambda_{k}}{2}-s_{k}\right), k=1,2$,

$\Lambda \in T^{2}, t \in T^{2}$.

Expressing all integrals in the equation $\Delta_{\Lambda}^{2}(z)=0$ through $J^{\star}(z)$ and rearranging algebraically, we reduce the latter equation to the form

$$
\theta_{\Lambda}(z) J^{\star}(z)=\chi_{\Lambda}(z)
$$

where $\theta_{\Lambda}(z)$ is a fifth-order polynomial in $z$, and $\chi_{\Lambda}(z)$ is a lower-order polynomial in $z$. Analyzing Equation (18) outside the set $G_{\Lambda}^{2}$ and taking into account that the function $J^{\star}(z)$ with $z \notin\left[m_{\Lambda}^{2}, M_{\Lambda}^{2}\right]$ is monotonic, we can easily verify that the equation has no more than five solutions outside the set $G_{\Lambda}^{2}$.

We now consider the case of $v=3$. Let the full quasi-momentum have the form $\Lambda=\left(\Lambda_{1}, \Lambda_{2}, \Lambda_{3}\right)=\left(\Lambda_{0}, \Lambda_{0}, \Lambda_{0}\right)$. If the parameters $\Lambda_{0}, J$ and $J_{1}$ satisfy the conditions in Theorems 7-8, then statements similar to those in the theorems 
are true. Only one additional BS $\tilde{\tilde{\varphi}}$ appears, whose energy value is $\tilde{\tilde{z}}$. This energy level is twice degenerate and $\tilde{\tilde{z}}<m_{\Lambda}^{3}\left(\tilde{\tilde{z}}>M_{\Lambda}^{3}\right)$, if $J>J_{1}\left(J<J_{1}\right)$. This additional BS vanishes when $J=J_{1}$ because it is incorporated into the continuous spectrum.

To prove this, we note that in this case, the function $\Delta_{\Lambda}^{3}(z)$ has the form $\Delta_{\Lambda}^{3}(z)=\left[1-2\left(J-J_{1}\right) \int_{T^{3}} \frac{\left[\cos \left(\frac{\Lambda_{0}}{2}-t_{1}\right)-\cos \left(\frac{\Lambda_{0}}{2}-t_{2}\right)\right]^{2} \mathrm{~d} t_{1} \mathrm{~d} t_{2} \mathrm{~d} t_{3}}{h_{\Lambda}\left(t_{1}, t_{2}, t_{3}\right)-z}\right]^{2} \tilde{\Psi}_{\Lambda}(z), t \in T^{3}$, where

$\tilde{\Psi}_{\Lambda}(z)=\left\{1-4 J_{1} \int_{T^{3}} \frac{3+3 \cos \Lambda_{0}-2 \cos \frac{\Lambda_{0}}{2}\left(\sum_{i=1}^{v} \cos \left(\frac{\Lambda_{0}}{2}-t_{i}\right)\right)}{h_{\Lambda}\left(t_{1}, t_{2}, t_{3}\right)-z} \mathrm{~d} t\right\}$

$\times\left[1-4\left(J-J_{1}\right) \int_{T^{3}} \frac{\cos \left(\frac{\Lambda_{0}}{2}-t_{1}\right)\left[\sum_{i=1}^{3} \cos \left(\frac{\Lambda_{0}}{2}-t_{i}\right)-3 \cos \frac{\Lambda_{0}}{2}\right]}{h_{\Lambda}\left(t_{1}, t_{2}, t_{3}\right)-z} \mathrm{~d} t\right]$

$-48\left(J-J_{1}\right) J_{1} \int_{T^{3}} \frac{\left\{3+3 \cos \Lambda_{0}-2 \cos \frac{\Lambda_{0}}{2}\left[\sum_{i=1}^{3} \cos \left(\frac{\Lambda_{0}}{2}-t_{i}\right)\right]\right\} \cos \left(\frac{\Lambda_{0}}{2}-t_{1}\right)}{h_{\Lambda}\left(t_{1}, t_{2}, t_{3}\right)-z} \mathrm{~d} t_{1} \mathrm{~d} t_{2} \mathrm{~d} t_{3}$ $\times \int_{T^{3}} \frac{\cos \left(\frac{\Lambda_{0}}{2}-t_{1}\right)-\cos \frac{\Lambda_{0}}{2}}{h_{\Lambda}\left(t_{1}, t_{2}, t_{3}\right)-z} \mathrm{~d} t_{1} \mathrm{~d} t_{2} \mathrm{~d} t_{3}$.

Therefore the equation $\Delta_{\Lambda}^{3}(z)=0$ is equivalent to the equations

$$
\left[1-2\left(J-J_{1}\right) \int_{T^{3}} \frac{\left[\cos \left(\frac{\Lambda_{0}}{2}-t_{1}\right)-\cos \left(\frac{\Lambda_{0}}{2}-t_{2}\right)\right]^{2} \mathrm{~d} t_{1} \mathrm{~d} t_{2} \mathrm{~d} t_{3}}{h_{\Lambda}\left(t_{1}, t_{2}, t_{3}\right)-z}\right]^{2}=0
$$

and

$$
\tilde{\Psi}_{\Lambda}(z)=0 .
$$

It is easy see that Equation (19) has a unique double solution $z^{\prime}$ if $J \neq J_{1}$ and $z^{\prime}<m_{\Lambda}^{3}\left(z^{\prime}>M_{\Lambda}^{3}\right)$, if $J>J_{1}\left(J<J_{1}\right)$. Expressing all integrals in Equation (20) through $J^{\star}(z)=\int_{T^{3}} \frac{\mathrm{d} t_{1} \mathrm{~d} t_{2} \mathrm{~d} t_{3}}{h_{\Lambda}\left(t_{1}, t_{2}, t_{3}\right)-z}$, we obtain the equation

$$
\tilde{\eta}_{\Lambda}(z) J^{\star}(z)=\tilde{\theta}_{\Lambda}(z),
$$

where

$$
\tilde{\eta}_{\Lambda}(z)=\left(J-J_{1}\right) \tilde{z}^{2}+24\left(J+J_{1}\right)\left(J-2 J_{1}\right) \cos ^{2} \frac{\Lambda_{0}}{2} \tilde{z}+1152 J_{1}\left(J-2 J_{1}\right)^{2} \cos ^{4} \frac{\Lambda_{0}}{2},
$$


and $\tilde{\theta}_{\Lambda}(z)=24\left(J-2 J_{1}\right)\left(J-5 J_{1}\right) \cos ^{2} \frac{\Lambda_{0}}{2}-\left(J-J_{1}\right) \tilde{z}$. Here $\tilde{z}=z-24\left(J-2 J_{1}\right)$. If $\tilde{\eta}_{\Lambda}(z) \neq 0$, Equation (21) is, in turn, equivalent to the equation

$$
J^{\star}(z)=\frac{\tilde{\theta}_{\Lambda}(z)}{\tilde{\eta}_{\Lambda}(z)} .
$$

Analyzing Equation (22) outside the set $G_{\Lambda}^{3}$ and taking into account that the function $J^{\star}(z)$ for $z \notin G_{\Lambda}^{3}$, is monotonic, we prove the statements made above.

If $\Lambda \neq\left(\Lambda_{0}, \Lambda_{0}, \Lambda_{0}\right)$, the system has no more than seven BSs (taking the energy degeneration order into account), and there exist sets $R_{k}, k=\overline{0,7}$, of the parameters $J, J_{1}$, and $\Lambda$ such that in every set $R_{k}$ the system has exactly $k$ BSs. The energy values of these BSs lie outside the set $G_{\Lambda}^{3}$. When passing from one of these sets to another, either some additional BSs of the operator $\tilde{H}_{2}$ appear or some existing BSs vanish.

In this case, the function $\Delta_{\Lambda}^{3}(z)$ has the form $\Delta_{\Lambda}^{3}(z)=\operatorname{det} D_{\Lambda}^{3}(z)$, where

$$
D_{\Lambda}^{3}(z)=\left(\begin{array}{cccc}
a_{1} & a_{2} & a_{3} & a_{4} \\
b_{1} & b_{2} & b_{3} & b_{4} \\
c_{1} & c_{2} & c_{3} & a_{4} \\
d_{1} & d_{2} & d_{3} & d_{4}
\end{array}\right),
$$

and $\quad a_{1}=1-4 J_{1} \int_{T^{3}} \frac{g_{\Lambda}(s) \mathrm{d} s}{h_{\Lambda}(s)-z}, \quad a_{k+1}=-4\left(J-J_{1}\right) \int_{T^{3}} \frac{f_{\Lambda_{k}}\left(s_{k}\right) \mathrm{d} s}{h_{\Lambda}(s)-z}, k=1,2,3$;

$$
b_{1}=-4 J_{1} \int_{T^{3}} \frac{\eta_{\Lambda_{1}}\left(s_{1}\right) g_{\Lambda}(s) \mathrm{d} s}{h_{\Lambda}(s)-z}, \quad b_{2}=1-4\left(J-J_{1}\right) \int_{T^{3}} \frac{\eta_{\Lambda_{1}}\left(s_{1}\right) f_{\Lambda_{1}}\left(s_{1}\right) \mathrm{d} s}{h_{\Lambda}(s)-z} \text {, }
$$$$
b_{k+1}=-4\left(J-J_{1}\right) \int_{T^{3}} \frac{\eta_{\Lambda_{1}}\left(s_{1}\right) f_{\Lambda_{k}}\left(s_{k}\right) \mathrm{d} s}{h_{\Lambda}(s)-z}, k=2,3 ; \quad c_{1}=-4 J_{1} \int_{T^{3}} \frac{\eta_{\Lambda_{2}}\left(s_{2}\right) g_{\Lambda}(s) \mathrm{d} s}{h_{\Lambda}(s)-z} \text {, }
$$$$
c_{k+1}=-4\left(J-J_{1}\right) \int_{T^{3}} \frac{\eta_{\Lambda_{2}}\left(s_{2}\right) f_{\Lambda_{k}}\left(s_{k}\right) \mathrm{d} s}{h_{\Lambda}(s)-z}, k=1,3 \text {, }
$$$$
c_{3}=1-4\left(J-J_{1}\right) \int_{T^{3}} \frac{\eta_{\Lambda_{2}}\left(s_{2}\right) f_{\Lambda_{2}}\left(s_{2}\right) \mathrm{d} s}{h_{\Lambda}(s)-z}, d_{1}=-4 J_{1} \int_{T^{3}} \frac{\eta_{\Lambda_{3}}\left(s_{3}\right) g_{\Lambda}(s) \mathrm{d} s}{h_{\Lambda}(s)-z},
$$$$
d_{k+1}=-4\left(J-J_{1}\right) \int_{T^{3}} \frac{\eta_{\Lambda_{3}}\left(s_{3}\right) f_{\Lambda_{k}}\left(s_{k}\right) \mathrm{d} s}{h_{\Lambda}(s)-z}, k=1,2 \text {; }
$$

$d_{4}=1-4\left(J-J_{1}\right) \int_{T^{3}} \frac{\eta_{\Lambda_{3}}\left(s_{3}\right) f_{\Lambda_{3}}\left(s_{3}\right) \mathrm{d} s}{h_{\Lambda}(s)-Z}$. In these formulas

$g_{\Lambda}(s)=\sum_{k=1}^{3}\left[1+\cos \Lambda_{k}-2 \cos \frac{\Lambda_{k}}{2} \cos \left(\frac{\Lambda_{k}}{2}-s_{k}\right)\right]$,

$f_{\Lambda_{k}}\left(s_{k}\right)=\cos \left(\frac{\Lambda_{k}}{2}-s_{k}\right)-\cos \frac{\Lambda_{k}}{2}, k=1,2,3, \eta_{\Lambda_{k}}\left(s_{k}\right)=\cos \left(\frac{\Lambda_{k}}{2}-s_{k}\right), k=1,2,3,$.

$\Lambda \in T^{3}, t \in T^{3}$.

Expressing all integrals in the equation $\Delta_{\Lambda}^{3}(z)=0$ through $J^{\star}(z)$ and rearranging algebraically, we reduce this equation to the form 


$$
J^{\star}(z)=\frac{A_{\Lambda}(z)}{B_{\Lambda}(z)}
$$

where $B_{\Lambda}(z)$ is a seventh-order polynomial in $z$, and $A_{\Lambda}(z)$ is a lower-order polynomial in $Z$. Therefore, this equation has no more than seven solutions outside the set $G_{\Lambda}^{3}$.

For an arbitrary $v>3$ and $\Lambda=\left(\Lambda_{1}, \Lambda_{2}, \cdots, \Lambda_{v}\right)=\left(\Lambda_{0}, \Lambda_{0}, \cdots, \Lambda_{0}\right)$, if the parameters $J, J_{1}$ and $\Lambda_{0}$ satisfy the conditions in Theorems 7-8, statements similar to those in the theorems are true. In this situation, the operator $\tilde{H}_{2}$ with $J \neq J_{1}$ has only one additional BS. The energy $z$ of this additional BS is degenerate $(v-1)$ times. Moreover, $z<m_{\Lambda}^{v}\left(z>M_{\Lambda}^{v}\right)$, if $J>J_{1}\left(J<J_{1}\right)$. For all other values of the full quasi-momentum $\Lambda$ of the system, the operator $\tilde{H}_{2}$ has no more than $2 v+1$ BSs (taking the energy degeneration order into account) with the energy values lying outside the set $G_{\Lambda}^{v}$.

The proof of these statements is based on finding zeros of the determinants $\Delta_{\Lambda}^{v}(z)$ of the operators. Expressing all integrals in $\Delta_{\Lambda}^{v}(z)$ through $J^{\star}(z)$, we can bring the equation $\Delta_{\Lambda}^{v}(z)=0$ to the form

$$
J^{\star}(z)=\frac{C_{\Lambda}(z)}{D_{\Lambda}(z)},
$$

where $D_{\Lambda}(z)$ is a $(2 v+1)$ th-order polynomial in $z$ and $C_{\Lambda}(z)$ is also a polynomial in $z$ whose order (with respect to $D_{\Lambda}(z)$ ) is lower. Analysis of Equation (23) outside the set $G_{\Lambda}^{v}$ leads to the proof of the statements made above.

Theorem 9. Let $J=J_{1}$ and $v$ be an arbitrary number. Then the operator $\tilde{H}_{2}$ has no more than one BS, and the corresponding energy level $z<m_{\Lambda}^{v}$ is not degenerate.

Proof. If $J=J_{1}$, the relations

$h_{1 \Lambda}(x, t)=-4 J_{1} \sum_{i=1}^{v}\left[1-2 \cos \frac{\Lambda_{i}}{2} \cos \left(\frac{\Lambda_{i}}{2}-x_{i}\right)+\cos \Lambda_{i}\right]$,

$h_{\Lambda}(x)=-4 J_{1} \sum_{i=1}^{v}\left[1-\cos \frac{\Lambda_{i}}{2} \cos \left(\frac{\Lambda_{i}}{2}-x_{i}\right)\right]$ hold. Using the determinant

$\Delta_{\Lambda}^{v}(z)$ and solving the corresponding equation, we obtain the statement in Theorem 9.

\section{Structure of Essential Spectrum and Discrete Spectrum of Three-Magnon Systems}

We first determine the structure of the essential spectrum of the three-magnon system and then estimate the number of three-magnon BSs in this system. Comparing Formulas (8) and (11) and using tensor products of Hilbert spaces and tensor products of operators in Hilbert spaces [22], we can verify that the operator $\tilde{H}_{3 \Lambda}$ can be represented in the form

$$
\tilde{H}_{3 \Lambda}=\tilde{H}_{2 \Lambda_{1}} \otimes I+I \otimes\left(\tilde{H}_{2 \Lambda_{2}}+\tilde{H}_{2 \Lambda_{3}}\right)+K_{\Lambda},
$$


where $I$ is the unit operator in the space $\mathcal{H}_{1}$, and $\tilde{H}_{2 \Lambda_{1}}$ and $\tilde{H}_{2 \Lambda_{2}}$ and $\tilde{H}_{2 \Lambda_{3}}$ are the energy operator of two-magnon systems, and $K_{\Lambda}$ are finite-dimensional operator, $\Lambda=\lambda+\mu+\gamma, \Lambda_{1}=\lambda+\mu, \Lambda_{2}=\lambda+\gamma, \Lambda_{3}=\mu+\gamma$.

The spectrum of $A \otimes I+I \otimes B$, where $A$ and $B$ are densely defined bounded linear operators, was studied in [23] [24] [25]. Explicit formulas expressing $\sigma_{\text {ess }}(A \otimes I+I \otimes B)$ and $\sigma_{\text {disc }}(A \otimes I+I \otimes B)$ in terms of $\sigma(A), \sigma_{\text {disc }}(A), \sigma(B)$ and $\sigma_{\text {disc }}(B)$ were given in those papers: $\sigma_{\text {disc }}(A \otimes I+I \otimes B)$ $=\left\{\left(\sigma(A) \backslash \sigma_{\text {ess }}(A)\right)+\left(\sigma(B) \backslash \sigma_{\text {ess }}(B)\right)\right\} \backslash\left\{\left(\sigma_{\text {ess }}(A)+\sigma(B)\right) \cup\left(\sigma(A)+\sigma_{\text {ess }}(B)\right)\right\}$, $\sigma_{\text {ess }}(A \otimes I+I \otimes B)=\left(\sigma_{\text {ess }}(A)+\sigma(B)\right) \cup\left(\sigma(A)+\sigma_{\text {ess }}(B)\right)$. It is clear that $\sigma(A \otimes E+E \otimes B)=\left\{z_{1}+z_{2}: z_{1} \in \sigma(A), z_{2} \in \sigma(B)\right\}$.

Note that, what the problems of finite-rank perturbations for the compact operators be considered in the work [26] [27] [28].

The following theorems describe the structure of the essential spectrum of $\tilde{H}_{2 \Lambda_{1}} \otimes I+I \otimes\left(\tilde{H}_{2 \Lambda_{2}}+\tilde{H}_{2 \Lambda_{3}}\right)$.

Theorem 10. If $J=2 J_{1}$ and $v$ be arbitrary. Then the essential spectrum of the operator $\tilde{H}_{3 \Lambda}$ consists of the set of five points:

$\sigma_{\text {ess }}\left(\tilde{H}_{3 \Lambda}\right)=\left\{0,-2 J_{1},-4 J_{1},-(4 v+2) J_{1}-4 J_{1} \sum_{i=1}^{v} \cos \Lambda_{1, i}\right.$, and the inequality $\left.-2(4 v+2) J_{1}-4 J_{1} \sum_{i=1}^{v}\left(\cos \Lambda_{2, i}+\cos \Lambda_{3, i}\right)\right\}$

$4 \leq N \leq 8 v+4$ holds for the number $N$ of three-magnon BSs.

Proof. It can be seen from Theorem 5, that for $J=2 J_{1}$ the operator $\tilde{H}_{2}$ has exactly two BSs $\varphi_{1}$ and $\varphi_{2}$ (not taking the order of the energy degeneration into account) with the energy values $z_{1}=-2 J_{1}$, $z_{2}=-(4 v+2) J_{1}-4 J_{1} \sum_{i=1}^{v} \cos \Lambda_{1, i}$, while the continuous spectrum of the operator $\tilde{H}_{2}$ is consists of one point $z=0$, therefore the essential spectrum of operator $\tilde{H}_{3 \Lambda}$ is consists of points $z_{1}=0, z_{2}=-2 J_{1}, z_{3}=-4 J_{1}$, $z_{4}=-(4 v+2) J_{1}-4 J_{1} \sum_{i=1}^{v} \cos \Lambda_{1, i}$, $z_{5}=-2(4 v+2) J_{1}-4 J_{1} \sum_{i=1}^{v}\left(\cos \Lambda_{2, i}+\cos \Lambda_{3, i}\right)$, i.e. $\sigma_{\text {ess }}\left(\tilde{H}_{3 \Lambda}\right)=\left\{0,-2 J_{1},-4 J_{1},-(4 v+2) J_{1}-4 J_{1} \sum_{i=1}^{v} \cos \Lambda_{1, i}\right.$, $\left.-2(4 v+2) J_{1}-4 J_{1} \sum_{i=1}^{v}\left(\cos \Lambda_{2, i}+\cos \Lambda_{3, i}\right)\right\}$ . The operator $\tilde{H}_{2 \Lambda_{1}} \otimes I+I \otimes\left(\tilde{H}_{2 \Lambda_{2}}+\tilde{H}_{2 \Lambda_{3}}\right)$ has a eigenvalues to equally $z_{1}=-6 J_{1}$, $z_{2}=-(4 v+6) J_{1}-4 J_{1} \sum_{i=1}^{v} \cos \Lambda_{1, i}$, $z_{3}=-(8 v+6) J_{1}-4 J_{1} \sum_{i=1}^{v}\left(\cos \Lambda_{2, i}+\cos \Lambda_{3, i}\right)$, and $z_{4}=-(12 v+6) J_{1}-4 J_{1} \sum_{i=1}^{v}\left(\cos \Lambda_{1, i}+\cos \Lambda_{2, i}+\cos \Lambda_{3, i}\right)$. The operator $K_{\Lambda}$ is a finite rank operator, to rank is equal to $8 v$. Consequently, in this case the number of three-magnon BS $N \leq 8 v+4$.

Theorem 11. Let $\Lambda_{1}=\tilde{\pi}, \Lambda_{2}=\tilde{\pi}, \Lambda_{3}=\tilde{\pi}$ and $J \neq J_{1}$. Then the essential spectrum of the operator $\tilde{H}_{3 \Lambda}$ consists of the set of three points: 
$\sigma_{\text {ess }}\left(\tilde{H}_{3 \Lambda}\right)=\left\{24 v\left(J-2 J_{1}\right), 24 v\left(J-2 J_{1}\right)-4\left(J-J_{1}\right), 24 v\left(J-2 J_{1}\right)-2\left(J-J_{1}\right)\right\}$, and the inequality $1 \leq N \leq 8 v+1$ holds for the number $N$ of three-magnon BSs.

Proof. It can be seen from Theorem 6 , that for $\Lambda=\tilde{\pi}$ and $J \neq J_{1}$ the operator $\tilde{H}_{2}$ has a unique BS $\varphi$ with the energy value $z=8 v\left(J-2 J_{1}\right)-2\left(J-J_{1}\right)$, and this energy level is $v$-fold degenerate. The continuous spectrum of the operator $\tilde{H}_{2}$ is consists of point $z=8 v\left(J-2 J_{1}\right)$, therefore the essential spectrum of the operator $\tilde{H}_{3 \Lambda}$ consists of points $z_{1}=24 v\left(J-2 J_{1}\right), \quad z_{2}=24 v\left(J-2 J_{1}\right)-4\left(J-J_{1}\right)$, $z_{3}=24 v\left(J-2 J_{1}\right)-2\left(J-J_{1}\right)$, i.e. $\sigma_{\text {ess }}\left(\tilde{H}_{3 \Lambda}\right)=\left\{24 v\left(J-2 J_{1}\right), 24 v\left(J-2 J_{1}\right)-4\left(J-J_{1}\right), 24 v\left(J-2 J_{1}\right)-2\left(J-J_{1}\right)\right\}$. The operator $\tilde{H}_{2 \Lambda_{1}} \otimes I+I \otimes\left(\tilde{H}_{2 \Lambda_{2}}+\tilde{H}_{2 \Lambda_{3}}\right)$ has a eigenvalues equal to $z=24 v\left(J-2 J_{1}\right)-6\left(J-J_{1}\right)$. The operator $K_{\Lambda}$ has a finite rank operator, with rank to equal to $8 v$. Consequently, in this case, the number of three-magnon BS $N \leq 8 v+1$.

We let $a=8\left(J-2 J_{1}\right)\left(3-\cos \frac{\Lambda_{1}}{2}-\cos \frac{\Lambda_{2}}{2}-\cos \frac{\Lambda_{3}}{2}\right)$, $b=8\left(J-2 J_{1}\right)\left(3+\cos \frac{\Lambda_{1}}{2}+\cos \frac{\Lambda_{2}}{2}+\cos \frac{\Lambda_{3}}{2}\right)$, $c=8\left(J-2 J_{1}\right)\left(2-\cos \frac{\Lambda_{2}}{2}-\cos \frac{\Lambda_{3}}{2}\right), d=8\left(J-2 J_{1}\right)\left(2+\cos \frac{\Lambda_{2}}{2}+\cos \frac{\Lambda_{3}}{2}\right)$, $e=8\left(J-2 J_{1}\right)\left(1-\cos \frac{\Lambda_{1}}{2}\right), f=8\left(J-2 J_{1}\right)\left(1+\cos \frac{\Lambda_{1}}{2}\right)$.

Theorem 12. Let $v=1, \quad J<J_{1}$ and $\left.\Lambda_{1} \in\right] 0, \pi\left[, \Lambda_{2} \in\right] 0, \pi\left[, \Lambda_{3} \in\right] 0, \pi[$ or $\left.\Lambda_{1} \in\right] \pi, 2 \pi\left[, \Lambda_{2} \in\right] \pi, 2 \pi\left[, \Lambda_{3} \in\right] \pi, 2 \pi[$.

a) If $\cos \frac{\Lambda_{1}}{2}>-\frac{J-J_{1}}{2 J_{1}}, \cos \frac{\Lambda_{2}}{2}>-\frac{J-J_{1}}{2 J_{1}}, \cos \frac{\Lambda_{3}}{2}>-\frac{J-J_{1}}{2 J_{1}}$ or $\cos \frac{\Lambda_{1}}{2}<\frac{J-J_{1}}{2 J_{1}}, \quad \cos \frac{\Lambda_{2}}{2}<\frac{J-J_{1}}{2 J_{1}}, \quad \cos \frac{\Lambda_{3}}{2}<\frac{J-J_{1}}{2 J_{1}}, \quad$ then the essential spectrum of the operator $\tilde{H}_{3 \Lambda}$ consists of the union of five intervals:

$$
\begin{aligned}
& \sigma_{e s s}\left(\tilde{H}_{3 \Lambda}\right)=[a, b] \cup\left[z_{1}+c, z_{1}+d\right] \cup\left[z_{2}+c, z_{2}+d\right] \\
& \cup\left[z_{3}+z_{4}+e, z_{3}+z_{4}+f\right] \cup\left[z_{5}+z_{6}+e, z_{5}+z_{6}+f\right]
\end{aligned}
$$

and the inequality $4 \leq N \leq 8 v+4$ holds for the number $N$ of three-magnon BSs.

b) If $\cos \frac{\Lambda_{1}}{2} \leq-\frac{J-J_{1}}{2 J_{1}}, \cos \frac{\Lambda_{2}}{2} \leq-\frac{J-J_{1}}{2 J_{1}}, \cos \frac{\Lambda_{3}}{2} \leq-\frac{J-J_{1}}{2 J_{1}}$ or $\cos \frac{\Lambda_{1}}{2} \geq \frac{J-J_{1}}{2 J_{1}}, \quad \cos \frac{\Lambda_{2}}{2} \geq \frac{J-J_{1}}{2 J_{1}}, \quad \cos \frac{\Lambda_{3}}{2} \geq \frac{J-J_{1}}{2 J_{1}}, \quad$ then the essential spectrum of the operator $\tilde{H}_{3 \Lambda}$ consists of the union of three intervals: $\sigma_{e s s}\left(\tilde{H}_{3 \Lambda}\right)=[a, b] \cup\left[z_{1}+c, z_{1}+d\right] \cup\left[z_{2}+z_{3}+e, z_{2}+z_{3}+f\right]$, and the inequality $1 \leq N \leq 8 v+1$ holds for the number of three-magnon BSs $N$.

c) If $\cos \frac{\Lambda_{1}}{2}>-\frac{J-J_{1}}{2 J_{1}}, \cos \frac{\Lambda_{2}}{2}<\frac{J-J_{1}}{2 J_{1}}, \cos \frac{\Lambda_{3}}{2}<\frac{J-J_{1}}{2 J_{1}}$ or 
$\cos \frac{\Lambda_{1}}{2}<\frac{J-J_{1}}{2 J_{1}}, \cos \frac{\Lambda_{2}}{2}>\frac{J-J_{1}}{2 J_{1}}, \cos \frac{\Lambda_{3}}{2}>\frac{J-J_{1}}{2 J_{1}}$ or $\cos \frac{\Lambda_{1}}{2}<-\frac{J-J_{1}}{2 J_{1}}$,

$\cos \frac{\Lambda_{2}}{2}>\frac{J-J_{1}}{2 J_{1}}, \cos \frac{\Lambda_{3}}{2}>\frac{J-J_{1}}{2 J_{1}}$ or $\cos \frac{\Lambda_{1}}{2}>\frac{J-J_{1}}{2 J_{1}}, \cos \frac{\Lambda_{2}}{2}<\frac{J-J_{1}}{2 J_{1}}$,

$\cos \frac{\Lambda_{3}}{2}<\frac{J-J_{1}}{2 J_{1}}$, then the essential spectrum of the operator $\tilde{H}_{3 \Lambda}$ consists of the union of four intervals:

$\sigma_{e s s}\left(\tilde{H}_{3 \Lambda}\right)=[a, b] \cup\left[z_{1}+c, z_{1}+d\right] \cup\left[z_{2}+c, z_{2}+d\right] \cup\left[z_{3}+z_{4}+e, z_{3}+z_{4}+f\right]$, and the inequality $2 \leq N \leq 8 v+2$ holds for the number of three-magnon BSs $N$.

Theorem 13. Let $J=J_{1}$ and $\left.\Lambda_{1} \in\right] 0, \pi\left[, \Lambda_{2} \in\right] 0, \pi\left[, \Lambda_{3} \in\right] 0, \pi[$ or $\left.\Lambda_{1} \in\right] \pi, 2 \pi\left[, \Lambda_{2} \in\right] \pi, 2 \pi\left[, \Lambda_{3} \in\right] \pi, 2 \pi[$.

a) If $0<\Lambda_{1}<\alpha_{1}$ or $\alpha_{2}<\Lambda_{1}<2 \pi$, and $0<\Lambda_{2}<\alpha_{1}$ or $\alpha_{2}<\Lambda_{2}<2 \pi$, and $0<\Lambda_{3}<\alpha_{1}$ or $\alpha_{2}<\Lambda_{3}<2 \pi$, respectively, then the essential spectrum of the operator $\tilde{H}_{3 \Lambda}$ consists of the union of three intervals:

$\sigma_{\text {ess }}\left(\tilde{H}_{3 \Lambda}\right)=[a, b] \cup\left[z_{1}+c, z_{1}+d\right] \cup\left[z_{2}+z_{3}+e, z_{2}+z_{3}+f\right]$, and the inequality $1 \leq N \leq 8 v+1$ holds for the number of three-magnon BSs $N$.

b) If $\Lambda_{1} \in\left[\alpha_{1}, \pi[\cup] \pi, \alpha_{2}\left[\right.\right.$ and $\Lambda_{2} \in\left[\alpha_{1}, \pi[\cup] \pi, \alpha_{2}[\right.$ and

$\Lambda_{3} \in\left[\alpha_{1}, \pi[U] \pi, \alpha_{2}\left[\right.\right.$, then the essential spectrum of the operator $\tilde{H}_{3 \Lambda}$ consists of single interval: $\sigma_{\text {ess }}\left(\tilde{H}_{3 \Lambda}\right)=[a, b]$, and the inequality $0 \leq N \leq 8 v$ holds for the number of three-magnon BSs $N$. Here $\alpha_{1} \approx 100^{\circ}, \alpha_{2} \approx 260^{\circ}$.

c) If $0<\Lambda_{1}<\alpha_{1}$ or $\Lambda_{2} \in\left[\alpha_{1}, \pi[U] \pi, \alpha_{2}\left[\right.\right.$ or $\Lambda_{3} \in\left[\alpha_{1}, \pi[U] \pi, \alpha_{2}[\right.$, then the essential spectrum of the operator $\tilde{H}_{3 \Lambda}$ consists of the union of two intervals: $\sigma_{\text {ess }}\left(\tilde{H}_{3 \Lambda}\right)=[a, b] \cup\left[z_{1}+c, z_{1}+d\right]$, and the inequality $0 \leq N \leq 8 v$ holds for the number of three-magnon BSs $N$.

Theorem 14. Let $J_{1}<J<2 J_{1}$ and $\left.\Lambda_{1} \in\right] 0, \pi\left[, \Lambda_{2} \in\right] 0, \pi\left[, \Lambda_{3} \in\right] 0, \pi[$ or $\left.\Lambda_{1} \in\right] \pi, 2 \pi\left[, \Lambda_{2} \in\right] \pi, 2 \pi\left[, \Lambda_{3} \in\right] \pi, 2 \pi[$.

a) If $\cos \frac{\Lambda_{1}}{2} \leq \frac{J-J_{1}}{2 J_{1}}, \cos \frac{\Lambda_{2}}{2} \leq \frac{J-J_{1}}{2 J_{1}}, \cos \frac{\Lambda_{3}}{2} \leq \frac{J-J_{1}}{2 J_{1}}$ or $\cos \frac{\Lambda_{1}}{2} \geq-\frac{J-J_{1}}{2 J_{1}}, \quad \cos \frac{\Lambda_{2}}{2} \geq-\frac{J-J_{1}}{2 J_{1}}, \quad \cos \frac{\Lambda_{3}}{2} \geq-\frac{J-J_{1}}{2 J_{1}}$, then the essential spectrum of the operator $\tilde{H}_{3 \Lambda}$ consists of the union of five intervals: $\sigma_{\text {ess }}\left(\tilde{H}_{3 \Lambda}\right)=[a, b] \cup\left[z_{1}+c, z_{1}+d\right] \cup\left[z_{2}+c, z_{2}+d\right]$, and the inequality $\cup\left[z_{3}+z_{4}+e, z_{3}+z_{4}+f\right] \cup\left[z_{5}+z_{6}+e, z_{5}+z_{6}+f\right]$ ' $4 \leq N \leq 8 v+4$ holds for the number of three-magnon BSs $N$.

b) If $\cos \frac{\Lambda_{1}}{2}>\frac{J-J_{1}}{2 J_{1}}, \cos \frac{\Lambda_{2}}{2}>\frac{J-J_{1}}{2 J_{1}}, \cos \frac{\Lambda_{3}}{2}>\frac{J-J_{1}}{2 J_{1}}$ or $\cos \frac{\Lambda_{1}}{2}<-\frac{J-J_{1}}{2 J_{1}}, \cos \frac{\Lambda_{2}}{2}<-\frac{J-J_{1}}{2 J_{1}}, \cos \frac{\Lambda_{3}}{2}<-\frac{J-J_{1}}{2 J_{1}}$, then the essential spectrum of the operator $\tilde{H}_{3 \Lambda}$ consists of the union of seven intervals: $\sigma_{\text {ess }}\left(\tilde{H}_{3 \Lambda}\right)=[a, b] \cup\left[z_{1}+c, z_{1}+d\right] \cup\left[z_{2}+c, z_{2}+d\right]$

$\cup\left[z_{3}+c, z_{3}+d\right] \cup\left[z_{4}+z_{5}+e, z_{4}+z_{5}+f\right]$, and the inequality $\bigcup\left[z_{6}+z_{7}+e, z_{6}+z_{7}+f\right] \cup\left[z_{8}+z_{9}+e, z_{8}+z_{9}+f\right]$ 
$9 \leq N \leq 8 v+9$ holds for the number of three-magnon BSs $N$.

c) If $\cos \frac{\Lambda_{1}}{2} \leq \frac{J-J_{1}}{2 J_{1}}, \cos \frac{\Lambda_{2}}{2}>\frac{J-J_{1}}{2 J_{1}}, \cos \frac{\Lambda_{3}}{2}>\frac{J-J_{1}}{2 J_{1}}$ or $\cos \frac{\Lambda_{1}}{2} \geq-\frac{J-J_{1}}{2 J_{1}}, \quad \cos \frac{\Lambda_{2}}{2}<-\frac{J-J_{1}}{2 J_{1}}, \cos \frac{\Lambda_{3}}{2}<-\frac{J-J_{1}}{2 J_{1}}$ or $\cos \frac{\Lambda_{1}}{2} \geq-\frac{J-J_{1}}{2 J_{1}}$, $\cos \frac{\Lambda_{2}}{2}>\frac{J-J_{1}}{2 J_{1}}, \cos \frac{\Lambda_{3}}{2}>\frac{J-J_{1}}{2 J_{1}}$, then the essential spectrum of the operator $\tilde{H}_{3 \Lambda}$ consists of the union of six intervals:

$\sigma_{\text {ess }}\left(\tilde{H}_{3 \Lambda}\right)=[a, b] \cup\left[z_{1}+c, z_{1}+d\right] \cup\left[z_{2}+c, z_{2}+d\right] \cup\left[z_{3}+c, z_{3}+d\right]$ $\bigcup\left[z_{4}+z_{5}+e, z_{4}+z_{5}+f\right] \cup\left[z_{6}+z_{7}+e, z_{6}+z_{7}+f\right]$ and the inequality $6 \leq N \leq 8 v+6$ holds for the number of three-magnon BSs $N$.

Theorem 15. Let $2 J_{1}<J<3 J_{1}$ and $\left.\Lambda \in\right] 0, \pi[$ or $\Lambda \in] \pi, 2 \pi[$

a) If $\cos \frac{\Lambda_{1}}{2}>\frac{J-J_{1}}{2 J_{1}}, \cos \frac{\Lambda_{2}}{2}>\frac{J-J_{1}}{2 J_{1}}$, or $\cos \frac{\Lambda_{1}}{2}<-\frac{J-J_{1}}{2 J_{1}}$, $\cos \frac{\Lambda_{2}}{2}<-\frac{J-J_{1}}{2 J_{1}}$, then the essential spectrum of the operator $\tilde{H}_{3 \Lambda}$ consists of the union of five intervals: $\sigma_{\text {ess }}\left(\tilde{H}_{3 \Lambda}\right)=[a, b] \cup\left[z_{1}+c, z_{1}+d\right] \cup\left[z_{2}+c, z_{2}+d\right]$, $\cup\left[z_{3}+z_{4}+e, z_{3}+z_{4}+f\right] \cup\left[z_{5}+z_{6}+e, z_{5}+z_{6}+f\right]$ and the inequality $4 \leq N \leq 8 v+4$ holds for the number of three-magnon BSs $N$.

b) If $\cos \frac{\Lambda_{1}}{2} \leq \frac{J-J_{1}}{2 J_{1}}, \cos \frac{\Lambda_{2}}{2} \leq \frac{J-J_{1}}{2 J_{1}}$ or $\cos \frac{\Lambda_{1}}{2} \geq-\frac{J-J_{1}}{2 J_{1}}$, $\cos \frac{\Lambda_{2}}{2} \geq-\frac{J-J_{1}}{2 J_{1}}$, then the essential spectrum of the operator $\tilde{H}_{3 \Lambda}$ consists of the union of three intervals:

$\sigma_{\text {ess }}\left(\tilde{H}_{3 \Lambda}\right)=[a, b] \cup\left[z_{1}+c, z_{1}+d\right] \cup\left[z_{2}+z_{3}+e, z_{2}+z_{3}+f\right]$, and the inequality $1 \leq N \leq 8 v+1$ holds for the number of three-magnon BSs $N$.

c) If $\cos \frac{\Lambda_{1}}{2}>\frac{J-J_{1}}{2 J_{1}}, \cos \frac{\Lambda_{2}}{2} \leq \frac{J-J_{1}}{2 J_{1}}$, or $\cos \frac{\Lambda_{1}}{2}<-\frac{J-J_{1}}{2 J_{1}}$ $\cos \frac{\Lambda_{2}}{2} \geq-\frac{J-J_{1}}{2 J_{1}}$, then the essential spectrum of the operator $\tilde{H}_{3 \Lambda}$ consists of the union of four intervals:

$\sigma_{\text {ess }}\left(\tilde{H}_{3 \Lambda}\right)=[a, b] \cup\left[z_{1}+c, z_{1}+d\right] \cup\left[z_{2}+c, z_{2}+d\right] \cup\left[z_{3}+z_{4}+e, z_{3}+z_{4}+f\right]$, and the inequality $2 \leq N \leq 8 v+2$ holds for the number of three-magnon BSs $N$.

d) If $\cos \frac{\Lambda_{1}}{2} \leq \frac{J-J_{1}}{2 J_{1}}, \cos \frac{\Lambda_{2}}{2}>\frac{J-J_{1}}{2 J_{1}}$ or $\cos \frac{\Lambda_{1}}{2} \geq-\frac{J-J_{1}}{2 J_{1}}$, $\cos \frac{\Lambda_{2}}{2}<-\frac{J-J_{1}}{2 J_{1}}$, then the essential spectrum of the operator $\tilde{H}_{3 \Lambda}$ consists of the union of four intervals:

$\sigma_{e s s}\left(\tilde{H}_{3 \Lambda}\right)=[a, b] \cup\left[z_{1}+c, z_{1}+d\right] \cup\left[z_{2}+z_{3}+e, z_{2}+z_{3}+f\right]$, and the inequality $\cup\left[z_{4}+z_{5}+e, z_{4}+z_{5}+f\right]$

$2 \leq N \leq 8 v+2$ holds for the number of three-magnon BSs $N$. 
Theorem 16. Let $J=3 J_{1}$ and $\Lambda_{1} \neq 0, \Lambda_{2} \neq 0$. Then the essential spectrum of the operator $\tilde{H}_{3 \Lambda}$ consists of the union of three intervals: $\sigma_{\text {ess }}\left(\tilde{H}_{3 \Lambda}\right)=[a, b] \cup\left[z_{1}+c, z_{1}+d\right] \cup\left[z_{2}+z_{3}+e, z_{2}+z_{3}+f\right]$, and the inequality $1 \leq N \leq 8 v+1$ holds for the number of three-magnon BSs $N$.

Theorem 17. Let $J>3 J_{1}$ and $\Lambda_{1} \neq 0, \Lambda_{2} \neq 0$. Then the essential spectrum of the operator $\tilde{H}_{3 \Lambda}$ consists of the union of five intervals: $\sigma_{e s s}\left(\tilde{H}_{3 \Lambda}\right)=[a, b] \cup\left[z_{1}+c, z_{1}+d\right] \cup\left[z_{2}+c, z_{2}+d\right]$
$\cup\left[z_{3}+z_{4}+e, z_{3}+z_{4}+f\right] \cup\left[z_{5}+z_{6}+e, z_{5}+z_{6}+f\right]$ $4 \leq N \leq 8 v+4$ holds for the number of three-magnon BSs $N$.

If $\Lambda_{1}$ and $\Lambda_{2}$ and $\Lambda_{3}$ has the form $\Lambda_{1}=\left(\Lambda_{1,1}, \Lambda_{1,2}, \cdots, \Lambda_{1, v}\right)=\left(\Lambda_{1,0}, \Lambda_{1,0}, \cdots, \Lambda_{1,0}\right)$, $\Lambda_{2}=\left(\Lambda_{2,1}, \Lambda_{2,2}, \cdots, \Lambda_{2, v}\right)=\left(\Lambda_{2,0}, \Lambda_{2,0}, \cdots, \Lambda_{2,0}\right)$, and $\Lambda_{3}=\left(\Lambda_{3,1}, \Lambda_{3,2}, \cdots, \Lambda_{3, v}\right)=\left(\Lambda_{3,0}, \Lambda_{3,0}, \cdots, \Lambda_{3,0}\right)$, then the essential spectrum of the operator $\tilde{H}_{3 \Lambda}$ is investigated to analogously to one-dimensional case.

In the essential spectrum of the operator $\tilde{H}_{3 \Lambda}$ is appear only two additional intervals and corresponding estimation for the number of three-magnon BSs, in the case of, when the operator $\tilde{H}_{2 \Lambda_{1}}$ and $\tilde{H}_{2 \Lambda_{2}}$ and $\tilde{H}_{2 \Lambda_{3}}$ has a correspondingly, correspondingly to equal to number a and b and $c$, BSs, that the estimation $a(b+c) \leq N \leq 8 v+a(b+c)$ changed to the estimation $(a+1)(b+c+1) \leq N \leq 8 v+(a+1)(b+c+1)$.

For arbitrary values $\Lambda_{1}$ and $\Lambda_{2}$ and $\Lambda_{3}$ and $v \geq 2$, the essential spectrum of the operator $\tilde{H}_{3 \Lambda}$ is consists of the union of no more that $(2 v+1)^{3}+1$ intervals, and the relation $N_{1} \leq N \leq 8 v+N_{1}$, holds for the number of three-magnon BSs $N$, where $0 \leq N_{1} \leq(2 v+1)^{3}$.

Theorem 18. If $J=J_{1}$ and the number $v$ be arbitrary. Then the essential spectrum of the operator $\tilde{H}_{3 \Lambda}$ consists of the union of no more then three intervals: $\sigma_{\text {ess }}\left(\tilde{H}_{3 \Lambda}\right)=[a, b] \cup\left[z_{1}+c, z_{1}+d\right] \cup\left[z_{2}+z_{3}+e, z_{2}+z_{3}+f\right]$, and the inequality $0 \leq N \leq 8 v+1$ holds for the number of three-magnon BSs $N$.

The cases, when $\Lambda_{1}=0$ or $\Lambda_{2}=0$ or $\Lambda_{3}=0$ investigated the similarly. Analogously is investigated essential spectrum and discrete spectrum of the operator $\tilde{H}_{3 \Lambda}$ for the other cases.

Obviously, that the case, when the of spin component $S^{z}$ take the value -1 coincide, with cases when spin component $S^{z}$ take the value 1 .

\section{Case, When $S_{m}^{Z} \varphi_{0}=0$}

We already say, what the spin component $S_{m}^{z}$ can by take on a value $-1,0,1$, i.e. can by $S_{m}^{z} \varphi_{0}=-\varphi_{0}$ or $S_{m}^{z} \varphi_{0}=0$ or $S_{m}^{z} \varphi_{0}=\varphi_{0}$.

Now we consider the case, when the of spin component $S^{z}$ take value 0 .

Hamiltonian of the system also has the form (3) and acts in the symmetrical Fo'ck space $\mathcal{H}$. In this case the vacuum vector $\varphi_{0}$ uniquely determined by the conditions: $S_{m}^{+} \varphi_{0}=0, S_{m}^{z} \varphi_{0}=0,\left\|\varphi_{0}\right\|=1$.

Theorem 19. The space $\mathcal{H}_{3}$ is invariant with respect of the operator $H$. The operator $H_{3}$ is a bounded self-adjoint operator. It generates the bounded 
self-adjoint operator $\bar{H}_{3}$, acting in the space $l_{2}\left(\left(Z^{v}\right)^{3}\right)$ according to the formula

$$
\begin{aligned}
& \left(\bar{H}_{3} f\right)(p, q, r) \\
& =-J \sum_{p, q, r, \tau}\left\{\left[\delta_{p, q+\tau}+\delta_{p+\tau, q}+\delta_{p, r+\tau}+\delta_{p+\tau, r}+\delta_{q+\tau, r}+\delta_{q, r+\tau}\right] f(p, q, r)\right. \\
& -\left(\frac{1}{2} \delta_{p-\tau, q}+\frac{1}{2} \delta_{p-\tau, r}\right) f(p-\tau, q, r)-\left(\frac{1}{2} \delta_{q-\tau, r}+\frac{1}{2} \delta_{p, q-\tau}\right) f(p, q-\tau, r) \\
& -\left(\frac{1}{2} \delta_{q, r-\tau}+\frac{1}{2} \delta_{p, r-\tau}\right) f(p, q, r-\tau)-\left(\frac{1}{2} \delta_{p+\tau, q}+\frac{1}{2} \delta_{p+\tau, r}\right) f(p+\tau, q, r) \\
& \left.-\left(\frac{1}{2} \delta_{q+\tau, r}+\delta_{p, q+\tau}\right) f(p, q+\tau, r)-\left(\frac{1}{2} \delta_{p, r+\tau}+\frac{1}{2} \delta_{q, r+\tau}\right) f(p, q, r+\tau)\right\} \\
& -J_{1} \sum_{p, q, r, \tau}\left\{\left(\delta_{p, q+\tau}+\delta_{p+\tau, q}+\delta_{p, r+\tau}+\delta_{p+\tau, r}+\delta_{q+\tau, r}+\delta_{q, r+\tau}+\frac{5}{2} \delta_{p, q+\tau} \delta_{q, r}\right.\right. \\
& \left.+\frac{5}{2} \delta_{q+\tau, r} \delta_{p, r}+\frac{5}{2} \delta_{q, r+\tau} \delta_{p, q}+\frac{5}{2} \delta_{p+\tau, r} \delta_{q, r}+\frac{5}{2} \delta_{q, r+\tau} \delta_{p, r}+\frac{5}{2} \delta_{q+\tau, r} \delta_{p, q}\right) f(p, q, r) \\
& -\left(\frac{1}{2} \delta_{p-\tau, q}+\frac{1}{2} \delta_{p-\tau, r}+\frac{3}{2} \delta_{q, r+\tau} \delta_{p, q}+\frac{3}{2} \delta_{q+\tau, r} \delta_{p, r}+\delta_{p, q+\tau} \delta_{q, r}\right) f(p-\tau, q, r) \\
& -\left(\frac{1}{2} \delta_{p+\tau, q}+\frac{1}{2} \delta_{q, r+\tau}+\frac{3}{2} \delta_{q, r+\tau} \delta_{p, q}+\frac{3}{2} \delta_{p+\tau, r} \delta_{q, r}+\delta_{q, r+\tau} \delta_{p, r}\right) f(p, q-\tau, r) \\
& -\left(\frac{1}{2} \delta_{p+\tau, r}+\frac{1}{2} \delta_{q+\tau, r}+\frac{3}{2} \delta_{q+\tau, r} \delta_{p, r}+\frac{3}{2} \delta_{p+\tau, r} \delta_{q, r}+\delta_{q+\tau, r} \delta_{p, q}\right) f(p, q, r-\tau) \\
& -\left(\frac{1}{2} \delta_{p+\tau, q}+\frac{1}{2} \delta_{p+\tau, r}+\frac{3}{2} \delta_{p+\tau, r} \delta_{q, r}+\frac{3}{2} \delta_{q+\tau, r} \delta_{p, q}+\delta_{q, r+\tau} \delta_{p, r}\right) f(p+\tau, q, r) \\
& -\left(\frac{1}{2} \delta_{p, q+\tau}+\frac{1}{2} \delta_{q+\tau, r}+\frac{3}{2} \delta_{p, r+\tau} \delta_{q, r}+\frac{3}{2} \delta_{q+\tau, r} \delta_{p, q}+\delta_{q+\tau, r} \delta_{p, r}\right) f(p, q+\tau, r) \\
& -\left(\frac{1}{2} \delta_{p, r+\tau}+\frac{1}{2} \delta_{q, r+\tau}+\frac{3}{2} \delta_{p, r+\tau} \delta_{q, r}+\frac{3}{2} \delta_{q, r+\tau} \delta_{p, r}+\delta_{q, r+\tau} \delta_{p, q}\right) f(p, q, r+\tau) \\
& +\delta_{q, r+\tau} \delta_{p, q} f(p-\tau, q-\tau, r)+\delta_{p, r} \delta_{q+\tau, r} f(p-\tau, q, r-\tau) \\
& +\delta_{p+\tau, r} \delta_{q, r} f(p, q-\tau, r-\tau)+\delta_{q+\tau, r} \delta_{p, q} f(p+\tau, q+\tau, r) \\
& +\delta_{q, r+\tau} \delta_{p, r} f(p+\tau, q, r+\tau)+\delta_{p, r+\tau} \delta_{q, r} f(p, q+\tau, r+\tau) \\
& +\left(\frac{1}{4} \delta_{p, r} \delta_{q+\tau, r}+\frac{1}{4} \delta_{p, r+\tau} \delta_{q, r}\right) f(p-\tau, q+\tau, r) \\
& +\left(\frac{1}{4} \delta_{p, q} \delta_{q, r+\tau}+\frac{1}{4} \delta_{p, r+\tau} \delta_{q, r}\right) f(p-\tau, q, r+\tau) \\
& +\left(\frac{1}{4} \delta_{p+\tau, r} \delta_{q, r}+\frac{1}{4} \delta_{p, r} \delta_{q, r+\tau}\right) f(p+\tau, q-\tau, r) \\
& +\left(\frac{1}{4} \delta_{p, q} \delta_{q+\tau, r}+\frac{1}{4} \delta_{q, r+\tau} \delta_{p, r}\right) f(p, q-\tau, r+\tau) \\
& +\left(\frac{1}{4} \delta_{p+\tau, r} \delta_{q, r}+\frac{1}{4} \delta_{p, q} \delta_{q+\tau, r}\right) f(p+\tau, q, r-\tau) \\
& \left.+\left(\frac{1}{4} \delta_{p, q} \delta_{q+\tau, r}+\frac{1}{4} \delta_{p, r} \delta_{q, r+\tau}\right) f(p, q+\tau, r)\right\} .
\end{aligned}
$$


where $\delta_{k, j}$ is the Kronecker symbol. The operator $H_{3}$ acts on the vector $\psi \in \mathcal{H}_{3}$ according to the formula

$$
H_{3} \psi=\sum_{p, q, r}\left(\bar{H}_{3} f\right)(p, q, r) S_{p}^{-} S_{q}^{-} S_{r}^{-} \varphi_{0} .
$$

Theorem 20. The Fourier transformation transforms the operator $\bar{H}_{3}$ into the bounded self-adjoint operator $\tilde{H}_{3}=\mathcal{F} \bar{H}_{3} \mathcal{F}^{-1}$, acting in the space $\tilde{\mathcal{H}}_{3}$ according to the formula

$$
\begin{aligned}
& \left(\tilde{H}_{3} f\right)(\lambda, \mu, \gamma) \\
& =-4\left(J+J_{1}\right)\left[\int_{T^{v}} h_{1}(\lambda, \mu, s) f(s, \lambda+\mu-s, \gamma) \mathrm{d} s\right. \\
& \left.+\int_{T^{v}} h_{1}(\lambda, \gamma, s) f(s, \mu, \lambda+\gamma-s) \mathrm{d} s+\int_{T^{v}} h_{1}(\mu, \gamma, s) f(\lambda, s, \mu+\gamma-s) \mathrm{d} s\right] \\
& +J \int_{T^{v}} \int_{T^{v}} h_{2}(\lambda, \mu, \gamma, s, t) f(s, t, \lambda+\mu+\gamma-s-t) \mathrm{d} s \mathrm{~d} t,
\end{aligned}
$$

where

$$
\begin{aligned}
& h_{1}(\lambda, \mu, s)=\sum_{i=1}^{v}\left[\cos \left(\lambda_{i}-s_{i}\right)+\cos \left(\mu_{i}-s_{i}\right)-\cos s_{i}-\cos \left(\lambda_{i}+\mu_{i}-s_{i}\right)\right], \\
& h_{2}(\lambda, \mu, \gamma, s, t) \\
& =\sum_{i=1}^{v}\left[4 \cos \left(s_{i}+t_{i}\right)+4 \cos \left(\lambda_{i}+\mu_{i}+\gamma_{i}-t_{i}\right)+4 \cos \left(\lambda_{i}+\mu_{i}+\gamma_{i}-s_{i}\right)\right. \\
& \quad+6 \cos \left(\mu_{i}+\gamma_{i}-s_{i}-t_{i}\right)+6 \cos \left(\lambda_{i}+\gamma_{i}-s_{i}-t_{i}\right)+6 \cos \left(\lambda_{i}+\mu_{i}-s_{i}-t_{i}\right) \\
& \quad+3 \cos \left(\lambda_{i}-s_{i}\right)+3 \cos \left(\lambda_{i}-t_{i}\right)+3 \cos \left(\mu_{i}-t_{i}\right)+3 \cos \left(\mu_{i}-s_{i}\right) \\
& \quad+3 \cos \left(\gamma_{i}-s_{i}\right)+3 \cos \left(\gamma_{i}-t_{i}\right)-4 \cos s_{i}-4 \cos t_{i}-4 \cos \left(\lambda_{i}+\mu_{i}-s_{i}\right) \\
& \quad-4 \cos \left(\lambda_{i}+\mu_{i}-t_{i}\right)-5 \cos \left(\mu_{i}-s_{i}-t_{i}\right)-5 \cos \left(\lambda_{i}-s_{i}-t_{i}\right) \\
& \quad-5 \cos \left(\gamma_{i}-s_{i}-t_{i}\right)-6 \cos \left(\mu_{i}+\gamma_{i}-s_{i}\right)-6 \cos \left(\lambda_{i}+\gamma_{i}-s_{i}\right) \\
& \left.\quad-5 \cos \left(\lambda_{i}+\mu_{i}+\gamma_{i}-s_{i}-t_{i}\right)\right] .
\end{aligned}
$$

The spectral properties of the considered energy operator of three-magnon systems in the isotropic ferromagnetic non-Heisenberg model are closely related to those of its two-magnon subsystems. We first study the spectrum and bound states of two-magnon subsystems.

Theorem 21. The space $\mathcal{H}_{2}$ is invariant with respect of the operator $H$. The operator $H_{2}$ is a bounded self-adjoint operator. It generates the bounded self-adjoint operator $\bar{H}_{2}$, acting in the space $l_{2}\left(\left(Z^{v}\right)^{2}\right)$ according to the formula

$$
\begin{aligned}
& \left(\bar{H}_{2} f\right)(p, q) \\
& =-\left(J+J_{1}\right) \sum_{p, q, \tau}\left\{\left[\delta_{p, q+\tau}+\delta_{p+\tau, q}\right] f(p, q)-\frac{1}{2} \delta_{p-\tau, q} f(p-\tau, q)\right. \\
& \left.-\frac{1}{2} \delta_{p, q-\tau} f(p, q-\tau)+\frac{1}{2} \delta_{p+\tau, q} f(p+\tau, q)+\frac{1}{2} \delta_{p, q+\tau} f(p, q+\tau)\right\} .
\end{aligned}
$$

The operator $H_{2}$ acts on the vector $\psi \in \mathcal{H}_{2}$ according to the formula

$$
H_{2} \psi=\sum_{p, q}\left(\bar{H}_{2} f\right)(p, q) S_{p}^{-} S_{q}^{-} \varphi_{0} .
$$

Theorem 22. The Fourier transformation transforms the operator $\bar{H}_{2}$ into 
the bounded self-adjoint operator $\tilde{H}_{2}=\mathcal{F} \bar{H}_{2} \mathcal{F}^{-1}$, acting in the space $\tilde{\mathcal{H}}_{2}$ according to the formula

$$
\left(\tilde{H}_{2} f\right)(x, y)=\int_{T^{r}} h_{1}(x, y, s) f(s, x+y-s) \mathrm{d} s,
$$

where

$$
h_{1}(x, y, s)=-4\left(J+J_{1}\right) \sum_{i=1}^{v}\left[\cos \frac{x_{i}-y_{i}}{2}-\cos \frac{x_{i}+y_{i}}{2}\right] \cos \left(\frac{x_{i}+y_{i}}{2}-s_{i}\right) .
$$

Let the full quasi-momentum of the system $x+y=\Lambda$ be fixed. Let $L_{2}\left(\Gamma_{\Lambda}\right)$ be the space of functions that are quadratically integrable over the manifold $\Gamma_{\Lambda}=\{(x, y): x+y=\Lambda\}$. It is known [21] that the operator $\tilde{H}_{2}$ and space $\tilde{\mathcal{H}}_{2}$ can be expanded into the direct integrals $\tilde{H}_{2}=\oplus \int_{T^{v}} \tilde{H}_{2 \Lambda} \mathrm{d} \Lambda, \tilde{\mathcal{H}}_{2}=\oplus \int_{T^{v}} \tilde{\mathcal{H}}_{2 \Lambda} \mathrm{d} \Lambda$ of the operators $\tilde{H}_{2 \Lambda}$ and the spaces $\tilde{\mathcal{H}}_{2 \Lambda}$ such that the spaces $\tilde{\mathcal{H}}_{2 \Lambda}$ are invariant with respect to the operators $\tilde{H}_{2 \Lambda}$ and the operators $\tilde{H}_{2 \Lambda}$ act in the space $\tilde{\mathcal{H}}_{2 \Lambda}$ according to the formula

$$
\left(\tilde{H}_{2 \Lambda} f_{\Lambda}\right)(x)=\int_{T^{r}} h_{1 \Lambda}(x, t) f_{\Lambda}(t) \mathrm{d} t
$$

where $h_{1 \Lambda}(x, t)=h_{1}(x, \Lambda-x, t)$ and $f_{\Lambda}(x)=f(x, \Lambda-x)$.

Theorem 23. Let full quasi-momentum of the system $\Lambda$ by arbitrary. Then the operator $\tilde{H}_{2}$ has a unique BS $\varphi$ with the energy value $z=-2\left(J+J_{1}\right)$ and it is $v$-fold degenerated.

Let the full quasi-momentum of the system $x+y+z=\Lambda$ be fixed. Then the operator $\tilde{H}_{3}$ and space $\tilde{\mathcal{H}}_{3}$ can be expanded into the direct integrals $\tilde{H}_{3}=\oplus \int_{T^{v}} \tilde{H}_{3 \Lambda} \mathrm{d} \Lambda, \tilde{\mathcal{H}}_{3}=\oplus \int_{T^{\nu}} \tilde{\mathcal{H}}_{3 \Lambda} \mathrm{d} \Lambda$.

We now determine the structure of the essential spectrum of the three-magnon system and then estimate the number of three-magnon BSs in this system. Comparing Formulas (27) and (30) and using tensor products of Hilbert spaces and tensor products of operators in Hilbert spaces [22], we can verify that the operator $\tilde{H}_{3 \Lambda}$ can be represented in the form

$$
\tilde{H}_{3 \Lambda}=\tilde{H}_{2 \Lambda_{1}} \otimes I+I \otimes\left(\tilde{H}_{2 \Lambda_{2}}+\tilde{H}_{2 \Lambda_{3}}\right)+\tilde{K}_{\Lambda},
$$

where $I$ is the unit operator in the space $\tilde{\mathcal{H}}_{1}$, and $\tilde{K}_{\Lambda}$ are finite-dimensional operator (see (27)).

Theorem 24. Let full quasi-momentum of the system $\Lambda$ by arbitrary. Then the essential spectrum of the operator $\tilde{H}_{3 \Lambda}$ consists of the three points: $0, z_{1}$ and $z_{2}+z_{3}$, where $z_{1}$ and $z_{2}$ and $z_{3}$ is a eigenvalue of the operators $\tilde{H}_{2 \Lambda_{1}}$ and $\tilde{H}_{2 \Lambda_{2}}$ and $\tilde{H}_{2 \Lambda_{3}}$, correspondingly, and the inequality $1 \leq N \leq 8 v+1$ holds for the number of three-magnon BSs $N$.

The finding results shown the structure of essential spectra and discrete spectrum of three-magnon system, in the cases, when the component $S^{z}$ of spin is receive the value 1 and 0 , is strongly different one another.

\section{Conflicts of Interest}

The author declares no conflicts of interest regarding the publication of this paper. 


\section{References}

[1] Bethe, H.A. (1931) Eigenverte und eigenfunction der linearen Atom kette. Zeitschrift für Physik, 71, 205-226. https://doi.org/10.1007/BF01341708

[2] Fukuda, N. and Wortis, M. (1963) Bound States in the Spin Wave Problem. Journal of Physics and Chemistry of Solids, 24, 1675-1677. https://doi.org/10.1016/0022-3697(63)90115-X

[3] Wortis, M. (1963) Bound States of Two Spin Waves in the Heisenberg Ferromagnet. Physical Review, 132, 85-97. https://doi.org/10.1103/PhysRev.132.85

[4] Majumdar, C.K. (1969) Bound States of Two Spin Waves in the Heisenberg Ferromagnet with Nearest and Next Nearest Neighbours Interactions. Journal of Mathematical Physics, 132, 85.

[5] Ono, I., Mikado, S. and Oguchi, T. (1971) Two-Magnon Bound States in a Linear Heisenberg Chain with Nearest and Next Nearest Neighbours Interactions. Journal of the Physical Society of Japan, 30, 358-366. https://doi.org/10.1143/JPSJ.30.358

[6] Gochev, I.G. (1973) Two-Magnon States in a One-Dimensional Heisenberg Models whit Second Nearest Neighbours Interactions. Theoretical and Mathematical Physics, 15, 402-406. https://doi.org/10.1007/BF01028269

[7] Gochev, I.G. (1972) Bound States of Magnon Systems in a Linear Anisotropic Chains. Journal of Experimental and Theoretical Physics, 34, 892.

[8] Tashpulatov, S.M. (1996) Investigation of the Energy Operator Spectrum for a Two-Magnon System in a One-Dimensional Anisotropic Heisenberg Ferromagnet with Second Neighbor Interactions. Theoretical and Mathematical Physics, 10, 544-549. https://doi.org/10.1007/BF02071461

[9] Shrödinger, E. (1941) Exchange and Spin. Proceedings of the Royal Irish Academy, $48,39$.

[10] Micnas, R. (1974) Heisenberg Ferromagnet with Biquadratic Exchange in the Random Phase Approximation. Physica Status Solidi, 66, 75-78. https://doi.org/10.1002/pssb.2220660253

[11] Brown, A.A. (1971) Heisenberg Ferromagnet with Biquadratic Exchange. Physical Review B, 4, 115-121. https://doi.org/10.1103/PhysRevB.4.115

[12] Chen, H.H. and Levy, P. (1971) Quadrupole Phase Transitions in Magnetic Solids. Physical Review Letters, 27, 1383-1385. https://doi.org/10.1103/PhysRevLett.27.1383

[13] Pink, D.A. and Ballard, R. (1974) Effect of Biquadratic Exchange. Canadian Journal of Physics, 52, 33-39. https://doi.org/10.1139/p74-005

[14] Pink, D.A. and Tremblay, P. (1972) Effect of Biquadratic Exchange upon Ferromagnetic Two-Magnon Bound States. Canadian Journal of Physics, 50, 1728-1735. https://doi.org/10.1139/p72-234

[15] Tashpulatov, S.M. (1996) Investigation of the Energy Operator Spectrum of the Two-Magnon System in a One-Dimensional Spin $s=1$ Non-Heisenberg Ferromagnet with Nearest and Second-Nearest Neighbor Interactions. Theoretical and Mathematical Physics, 107, 620-628. https://doi.org/10.1007/BF02071375

[16] Tashpulatov, S.M. (1996) Two-Magnon States in a One-Dimensional Non-Heisenberg Ferromagnet with Spin One and Nearest-Neighbor Coupling. Theoretical and Mathematical Physics, 107, 629-634. https://doi.org/10.1007/BF02071376

[17] Tashpulatov, S.M. (2000) Spectra and Bound States of the Energy Operator of Two-Magnon Systems in a Non-Heisenberg Ferromagnet with Spin One and Near- 
est-Neighbor Coupling. Theoretical and Mathematical Physics, 125, 1539-1551. https://doi.org/10.1007/BF02551013

[18] Van Himbergen, J.E. and Tjon, J.A. (1976) Three-Magnon Bound States in the Two-Dimensional Isotropic and Anisotropic Heisenberg Ferromagnet. Physica A, 82, 389-416. https://doi.org/10.1016/0378-4371(76)90015-7

[19] Tashpulatov, S.M. (2010) Spectrum of the Energy Operator of Two-Magnon Systems in the Isotropic Heisenberg Ferromagnet Model with Impurity. Theoretical and Mathematical Physics, 164, 1222-1229. https://doi.org/10.1007/s11232-010-0104-5

[20] Tashpulatov, S.M. (2013) Spectrum of Two-Magnon Non-Heisenberg Ferromagnetic Model of Arbitrary Spin with Impurity. Journal of Mathematical Physics, Analysis, Geometry, 9, 239-265.

[21] Neimark, M.A. (1968) Normed Rings. Nauka, Moscow.

[22] Reed, M. and Simon, B. (1972) Methods of Modern Mathematical Physics, Vol. 1, Functional Analysis. Academic Press, New York.

[23] Ichinose, T. (1978) Spectral Properties of Tensor Products of Linear Operators. Transactions of the American Mathematical Society, 235, 75-113. https://doi.org/10.1090/S0002-9947-1978-0472915-2

[24] Ichinose, T. (1978) Spectral Properties of Tensor Products of Linear Operators. 2: The Approximate Point Spectrum and Kato Essential Spectrum. Transactions of the American Mathematical Society, 237, 223-254. https://doi.org/10.1090/S0002-9947-1978-0472915-2

[25] Ichinose, T. (1982) Tensor Products of Linear Operators. Spectral Theory. Banach Center Publications, PWN-Polish Scientific Publishers, Warsaw, Vol. 8, 294-300.

[26] Hochstadt, H. (1973) One Dimensional Perturbations of Compact Operators. Proceedings of the American Mathematical Society, 37, 465-467. https://doi.org/10.1090/S0002-9939-1973-0310681-2

[27] Vasudeva, H. (1976) One Dimensional Perturbations of Compact Operators. Proceedings of the American Mathematical Society, 57, 58-60. https://doi.org/10.1090/S0002-9939-1976-0445318-8

[28] Savchenko, S.V. (2004) On the Change in the Spectral Properties of a Matrix under Perturbations of Sufficiently Low Rank. Functional Analysis and Its Appilications, 38, 69-71. https://doi.org/10.1023/B:FAIA.0000024871.00388.4c 